\title{
Partial Turbulence Simulation Method for Predicting Peak Wind Loads on Small
}

\section{Structures and Building Appurtenances}

\author{
Maryam Asghari Mooneghi ${ }^{\mathrm{a}}$, Peter Irwin ${ }^{\mathrm{b}}$, Arindam Gan Chowdhury ${ }^{\mathrm{c}}$ \\ ${ }^{a} \mathrm{PhD}$, Advanced Technology and Research, Arup, San Francisco, CA, USA \\ ${ }^{\mathrm{b}}$ Professor of Practice, ${ }^{\mathrm{c}}$ Corresponding Author, Associate Professor, Civil and Environmental Engineering/ \\ International Hurricane Research Center, Florida International University, Miami, FL, USA \\ 므aryam.asghari@arup.com, ${ }^{\mathrm{b}}$ peairwi@fiu.edu, ${ }^{\mathrm{c}}$ chowdhur@fiu.edu,
}

\begin{abstract}
Large-scale wind tunnel testing is preferred for small structures and building appurtenances for maintaining modeling accuracy and minimizing Reynolds number effects. In these circumstances the ability to obtain a large enough turbulence integral scale is usually compromised by the limited dimensions of the wind tunnel. So, it is not normally possible to fully simulate the low frequency end of the turbulence spectrum. In this paper the approach is taken of dividing the turbulence into two distinct statistical processes, one at high frequencies which can be simulated in the wind tunnel, and one at low frequencies which can be treated in post-test analysis using the assumptions of quasi-steady theory. In this Partial Turbulence Simulation (PTS) method the contribution of both the high and low frequency turbulence on the wind loads on the structures is included by using the probability of load from each of the two processes, with one part coming from the wind tunnel data representing the high frequency component and the remainder from the assumed probability distribution (taken in this paper as Gaussian for generic boundary layer flow) of the missing low frequency component. The two processes are approximated as independent of each other. The efficacy and validity of the method and its various assumptions are assessed by comparing predicted local peak pressure coefficients from tests on large scale models of the Silsoe cube and Texas Tech University (TTU) building in the Wall of Wind facility at Florida International University (FIU) with the corresponding full-scale data. Generally good agreement was found between the model results and full scale, particularly when comparing the highest overall peak pressure coefficients. These results, although limited to peak local pressures on the two test buildings for which good full scale data are available, are encouraging and invite further experiments to explore the range of applicability of the PTS method. This method, although developed in the Wall of Wind facility at FIU, can be equally used in conventional boundary layer wind tunnels and has the potential to enhance the ability of existing boundary layer wind tunnel facilities to predict full scale wind loads.
\end{abstract}

Keywords: Low-rise Building, Partial Turbulence Simulation, Pressure Coefficient, Wind Load

\section{Introduction}

Boundary layer wind tunnel testing has been generally accepted as a useful tool for evaluating wind loads on structures. For tall buildings the model scales used are typically in the range of 1:300 to 1:600. At these scales it is possible in typical sized wind tunnels to simulate the wind velocity profile, turbulence intensity and turbulence integral scale such that all represent well the corresponding values at full scale. However, for small structures like 
low-rise buildings, and for building appurtenances, the model scales used are often larger, in the range of 1:10 to 1:100 in order to keep Reynolds numbers high enough to avoid adverse scale effects (Kargarmoakhar et al, 2015), better replicate the effects of architectural features, and to be able to obtain adequate spatial resolution of pressures taps. For some tests even larger scales are desirable. At these large model scales the ability to obtain a large enough turbulence integral scale in the wind tunnel is compromised by the limited dimensions of the wind tunnel (Stathopoulos and Surry, 1983). As a result many of the model tests on these structures have been undertaken with less than ideal simulation of the turbulence integral scale.

Both small-scale and large-scale turbulence play an important role in the development of the peak wind pressures. The small scale turbulence interacts directly with the turbulent shear layers and vortices that originate at the edges of the roof and walls and then pass over the roof and wall surfaces. The configurations and strengths of these shear layers and vortices, which directly affect the suctions on the building surfaces, can be significantly altered by the small scale turbulence. Therefore accurate simulation of high frequency turbulence is necessary in order to correctly model flow separation and reattachment (Asghari Mooneghi, 2014; Asghari Mooneghi et al, 2014; Banks, 2011; Irwin, 2009; Kopp and Banks, 2013; Kumar and Stathopoulos, 1998; Melbourne, 1980; Richards et al, 2007; Saathoff and Melbourne, 1997; Tieleman, 2003; Yamada and Katsuchi, 2008). The large scale turbulence tends to cause low frequency fluctuations in the oncoming wind speed and direction, which then cause low frequency movements and changes in strength of the shear layers and vortices. Notionally these low frequency fluctuations may be treated using the quasi-steady approximation, i.e. as if they are similar to changes in mean wind speed and direction, and this approach is explored in this paper.

While the focus in this paper is on buildings it is worth noting that similar issues of turbulence simulation have also arisen in sectional model testing of bridges, typically done at scales in the range 1:30 to 1:100. The use of partial simulation of turbulence for bridges can in fact be traced back to the 1980's. Davenport and King (1984) used grid generated turbulence, deficient at low frequencies, on sectional models so as to include the aerodynamic effects of turbulence. The missing low frequency component of turbulence was compensated for analytically, essentially using quasi-steady assumptions. Also, Wardlaw et al (1983) reported results of experiments on the effect of grid generated turbulence on bridge decks. The integral scales were less than at full scale implying that only the high frequency component was simulated. Irwin (1998) described the requirement for spectrum matching for bridges (essentially Equation 18 of the present paper) when using partial turbulence simulation. It was applied in sectional model tests of 
the Second Severn Bridge (Macdonald et al, 2002) and much better agreement with full scale observations of vortex excitation was obtained when partial turbulence simulation was used as compared to smooth flow.

Comparison with full-scale data is the ultimate test of the validity of scale model testing in wind tunnels. Studies by a number of researchers on comparisons of mean pressure coefficients have demonstrated good agreement in many cases. However, discrepancies have been observed for the peak suction pressures, mainly in regions of (1) flow separation near the leading edges of the roof, and (2) conical vortices near the windward roof corners for oblique wind azimuth angles (Cheung et al, 1997; Cochran and Cermak, 1992; Okada and Ha, 1992; Surry, 1991). In wind tunnel studies on the Texas Tech University (TTU) test building (Lin et al, 1995; Okada and Ha, 1992; Surry, 1991; Tieleman et al, 1996) good agreement between the laboratory and field data was found for mean pressures. However, the agreement for the peak and root-mean-square (RMS) point pressures was found to be less satisfactory at critical locations in the roof corner region. A similar result was obtained by Richards et al (2007) when comparing 1:40 scale wind tunnel results with full-scale data on the Silsoe cube. One of the main reasons of this discrepancy was attributed to mismatches in the turbulence spectrum, i.e. not enough content at low frequencies and too much at high frequencies. Recent studies suggest that in addition to properly simulating the longitudinal turbulence intensity (Hillier and Cherry, 1981; Melbourne, 1980, 1993; Saathoff and Melbourne, 1989), the simulation of lateral (Letchford and Mehta, 1993; Richards et al, 2015; Tieleman, 2003; Tieleman et al, 1996; Zhao, 1997) and vertical (Wu et al, 2001) turbulence intensities can affect results for the peak-suction pressures especially near a roof corner. If the overall longitudinal and transverse turbulence intensities are matched on the model, but the integral scale is too small, then the high frequency part of the spectrum has too much power. To correctly match the spectrum at high frequencies in this situation, it is required that the model turbulence intensity be smaller than at full scale (Asghari Mooneghi et al, 2015; Banks, 2011; Richards et al, 2007; Yamada and Katsuchi, 2008) but then the question arises as to how to account for the missing low frequency content.

This paper presents a theoretical and experimental approach to account for the effects of the low frequency fluctuations in the wind flow. It assumes that all the effects of the high frequency fluctuations are captured by measurements in a wind flow that has the high frequency part of the turbulence spectrum at the right energy level. The effects of low frequency fluctuations are then accounted for analytically using quasi-steady theory. As already noted quasi-steady theory has been used before to examine the buffeting response of bridges (Davenport and King, 1984). It has also been used by Diana et al (1999) to investigate the turbulence effects on flutter. In their 
analytical model the total response was decomposed into components with different frequencies in order to incorporate frequency dependent characteristics. Chen et al (2000) developed a time domain analysis framework for predicting the flutter and buffeting responses of bridges under turbulent winds which included the nonlinear aerodynamics with respect to the effective angle of incidence. In their approach, the turbulence was again divided into low frequency and high frequency components with the low frequency turbulence being expressed using the quasi-steady approximation.

In the context of testing small structures ASCE/SEI 49-12 (2012) discusses the need for additional interpretation of wind tunnel data when the complete spectrum of wind turbulence is not simulated, but does not define a methodology. An aim of the present research is to explore methodology for achieving an appropriate interpretation. There are two versions of the presently proposed method. The simplified version is called the "Partial Turbulence Simulation (PTS)" method in which just the effect of missing low frequency longitudinal turbulence is considered. The extended version of PTS is called 3 Dimensional Partial Turbulence Simulation (3DPTS) which simulates the additional effects of the missing low frequency lateral and vertical fluctuations. The 3DPTS method requires a number of additional tests using small incremental wind azimuth and pitch angles centered on the mean wind vector of interest. To assess the accuracy of both the PTS and 3DPTS methods, pressures on large-scale models of the Silsoe cube (Richards and Hoxey, 2012) and TTU building (Levitan and Mehta, 1992a and 1992b) were measured in the Wall of Wind (WOW) facility at Florida International University (FIU) with only the high frequency part of the turbulence spectrum simulated experimentally. Then the effects of the missing low frequency fluctuations were incorporated analytically by analyzing the experimental data using the PTS and 3DPTS methods to predict the full

112 scale pressure coefficients. The final results were compared with the pressure coefficients obtained from field data on the respective prototypes in the real atmospheric flow with full turbulence spectrum.

\section{Theory}

\subsection{Small and large scale turbulence}

117 The aerodynamic behavior of a bluff structure such as a building is governed by the state of flow separation around 118 it which is greatly affected by the oncoming flow turbulence. As indicated earlier, it is known that small-scale turbulence interacts in important ways with the shear layers and vortices cast off from a body immersed in turbulent air flow. On the other hand, very large-scale turbulent eddies, much bigger than the body, can be expected to have a 
somewhat similar effect to a change in the mean flow velocity vector. This suggests that if a sufficient range of the small-scale turbulence can be simulated in a wind tunnel then it might be possible to include the large-scales later in post-test analysis using quasi-steady assumptions.

\subsection{Equilibrium of small scale turbulence}

Townsend (1961) described the existence in fluid flow near a solid boundary of an equilibrium layer within which local turbulence energy generation and dissipation are in approximate balance and far outweigh diffusion and advection of turbulence energy. One would expect that near enough to the ground the natural wind would also approximate an equilibrium layer. Within such a layer one would expect small scale turbulence to rapidly adjust to changes caused by large-scale, low frequency turbulence. Experimental evidence for this in flow over a smooth surface is provided by Hutchins and Marusic (2007). The non-dimensional Reynolds stresses and mean velocity profile should converge to universal values consistent with the universal law of the wall, provided that the averaging time over which they are determined corresponds to a wave length large compared to the height above ground. This behavior has been exploited by measurement devices such as the Irwin Sensor (Irwin, 1981) that rely on the existence of the law of the wall for their calibration. Irwin demonstrated that these devices can measure not only mean flow velocities but also velocity fluctuations caused by turbulent eddies provided these eddies are much larger than the height of the sensor or probe. In an analogous way for a low building or small structure of height $H$, if the turbulent eddies are an order of magnitude greater in size than $H$, it appears possible that their effects may be able to be approximated as quasi-steady variations.

Based on the above discussion we assume that the small scales of turbulence rapidly reach an equilibrium state when changes are imposed by large scale turbulence, and for convenience we consider the total velocity fluctuations as being made up of two parts, a low frequency (large scale) part and a high frequency (small scale) part. The question of where the dividing line occurs will be deferred to the next section. It is acknowledged that rapid equilibrium is an approximation and that in reality there is no such sharp dividing line between low and high frequencies. However, it is nonetheless of interest to see where these theoretical assumptions lead and how well they approximate the real flow behavior.

As explained previously, in the simplified version of the theory (PTS) only the effects of missing low frequency longitudinal velocity fluctuations are incorporated analytically while the possible effects of low frequency changes 
in flow angle are ignored. In the 3DPTS approach which is discussed later the effects of low frequency lateral and vertical fluctuations are included.

\subsection{PTS Method}

Considering just the longitudinal component of turbulence, we express the total velocity $\widetilde{U}$ at any given instant as:

$\widetilde{U}=U+u_{L}+u_{H}$

where $U$ is the mean flow velocity, $u_{L}$ is the part of the fluctuating velocity contributed by the low frequency end of the spectrum and $u_{H}$ is the part contributed by the high frequency end. In a partial turbulence simulation test, where in the experiment we only simulate the high frequency part of the spectrum, the mean velocity of the test, $U_{p s}$, is effectively the mean speed $U$ corresponding to an atmospheric flow (with full spectrum) plus whatever the low frequency gust component $u_{L}$ is at the time, as illustrated in Fig. 1.

$U_{p s}=U+u_{L}$

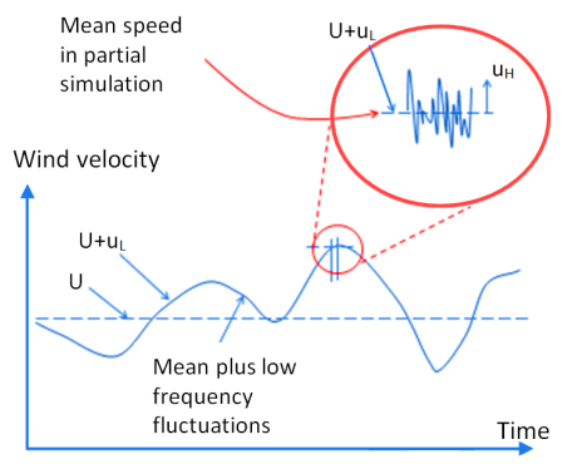

Fig.1. Illustration of mean flow velocity, low frequency and high frequency fluctuations

The overall variance of the turbulence when the full spectrum is present is:

$\sigma_{u}^{2}=\overline{\overline{\left(u_{L}+u_{H}\right)^{2}}}$

where the double over-bar denotes the mean value over a long enough time to attain statistical stability of the low frequency turbulence quantities such as the RMS of the low frequency velocity fluctuations, $\sigma_{u L}$. Since in the partial simulation the mean velocity is $U+u_{L}$, our measured partial simulation turbulence intensity, called $I_{u H}$ here, is:

$I_{u H}=\frac{\sqrt{\overline{u_{H}^{2}}}}{U+u_{L}}=\frac{\sigma_{u H}}{U+u_{L}}$

where the single over-bar denotes mean values over a long enough time to attain statistical stability of high frequency quantities such as $\sigma_{u H}$. At this point we bring in the rapid equilibrium assumption, which is that $I_{u H}$ is a constant as far as the low frequency flow variations are concerned. If a low frequency gust occurs, i.e. $u_{L}$ increases, 
then $\sigma_{u H}$ adjusts quickly to the new amount of energy being fed in from the large scale turbulence. In other words the high frequency turbulence rapidly attains a new equilibrium with increased energy, with $I_{u H}$ remaining constant. Using Eq. (4), we may express $u_{H}$ in the following form.

$u_{H}=\frac{u_{H}}{\sigma_{u H}} \sigma_{u H}=\frac{u_{H}}{\sigma_{u H}}\left(U+u_{L}\right) I_{u H}$

In this equation the ratio $\frac{u_{H}}{\sigma_{u H}}$ is a variable that fluctuates rapidly at high frequency but the fluctuations are not correlated with the low frequency fluctuations of $u_{L}$. The only link with low frequency fluctuations is through the term $\left(U+u_{L}\right)$ which is independent of high frequency fluctuations of $\frac{u_{H}}{\sigma_{u H}}$. Then Eq. (3) becomes:

$\sigma_{u}^{2}=\overline{\overline{\left(u_{L}+\left(U+u_{L}\right) I_{u H} \frac{u_{H}}{\sigma_{u H}}\right)^{2}}}=\overline{\overline{u_{L}^{2}+\left(U^{2}+2 U u_{L}+u_{L}^{2}\right) I_{u H}^{2} \frac{u_{H}^{2}}{\sigma_{u H}^{2}}+2 u_{L}\left(U+u_{L}\right) I_{u H} \frac{u_{H}}{\sigma_{u H}}}}$

Since the high frequency fluctuations of $\frac{u_{H}}{\sigma_{u H}}$ are assumed to be uncorrelated with the low frequency fluctuations of $u_{L}$, the mean values of cross-products $u_{L}\left(\frac{u_{H}}{\sigma_{u H}}\right), u_{L}^{2}\left(\frac{u_{H}}{\sigma_{u H}}\right)$ and $u_{L}\left(\frac{u_{H}}{\sigma_{u H}}\right)^{2}$ are zero. Also we can ignore the higher order term $\overline{\overline{I_{u H}^{2} u_{L}^{2}\left(\frac{u_{H}}{\sigma_{u H}}\right)^{2}}}$ as being very small compared to the other terms. Then, noting that the mean of $\left(\frac{u_{H}}{\sigma_{u H}}\right)^{2}$ is 1 , Eq. (3) becomes:

$\sigma_{u}^{2}=\overline{u_{L}^{2}}+U^{2} I_{u H}^{2}$

Therefore, in terms of turbulence intensities, the total turbulence intensity $I_{u}$ is given by

$I_{u}=\frac{\sigma_{u}}{U}=\sqrt{I_{u L}^{2}+I_{u H}^{2}}$

where $I_{u L}=\frac{\sigma_{u L}}{U}$.

From Eq. (8) it is observed that if we have done a partial turbulence simulation with turbulence intensity $I_{u H}$ then the intensity of the missing low frequency intensity is given by:

$I_{u L}=\sqrt{I_{u}^{2}-I_{u H}^{2}}$

Similar formulas can be written for lateral and vertical components of the wind velocity components.

\subsection{Determination of dividing frequency $n_{c}$ between low and high frequencies}

When we do a partial simulation we know our turbulence intensity is $I_{u H}$ and that our missing low frequency turbulence intensity is $I_{u L}$, as given by Eq. (9). Equation (9) can also be used to show that:

$\left(\frac{I_{u H}}{I_{u}}\right)^{2}=1-\left(\frac{I_{u L}}{I_{u}}\right)^{2}=\frac{\sigma_{u}^{2}-\sigma_{u L}^{2}}{\sigma_{u}^{2}}=\int_{n_{c}}^{\infty} \frac{S_{u}(n)}{\sigma_{u}^{2}} d n=\frac{U}{{ }^{{ } L_{u}}} \int_{\frac{c_{c}{ }^{x} L_{u}}{U}}^{\infty} \frac{s_{u}\left(\frac{n^{\times} L_{u}}{U}\right)}{\sigma_{u}^{2}} d\left(\frac{n^{\mathrm{x}} L_{u}}{U}\right)$ 
where $n=$ frequency, $S_{u}(n)=$ average power spectrum of $u$ over a long enough time to attain statistical stability of the whole spectrum, and $n_{c}$ is the "critical frequency" dividing the high and low frequency parts of the spectrum. Note that the longitudinal integral scale of the turbulence ${ }^{\mathrm{x}} L_{u}$ of the full spectrum is used here as a convenient length, along with the velocity $U$ for converting frequency to the non-dimensional form $\frac{n^{{ }^{x}} L_{u}}{U}$. The von Karman spectrum, which generally gives a good description of $S_{u}$, is:

$\frac{n S_{u}}{\sigma_{u}^{2}}=\frac{4 \frac{n^{\times} L_{u}}{U}}{\left(1+70.78\left(\frac{n^{\times} L_{u}}{U}\right)^{2}\right)^{5 / 6}}$

It can be noted that the maximum in the von Karman spectrum occurs at $n^{\mathrm{x}} L_{u} / U \approx 0.14$, which implies that the wave length $U / n$ of eddies at the maximum is about 7 times the integral scale ${ }^{\mathrm{x}} L_{u}$. Using Eq. (11) in Eq. (10) gives:

$\left(\frac{I_{u H}}{I_{u}}\right)^{2}=\int_{\eta_{c}}^{\infty} \frac{4}{\left(1+70.78 \eta^{2}\right)^{5 / 6}} d \eta$

where $\eta_{c}=\frac{n_{c}{ }^{\mathrm{x}} L_{u}}{U}$. In general, with the partial simulation approach we expect that we will be integrating over a range of frequencies where the second term in the denominator dominates. Thus we may simplify Eq. (12) to:

$\left(\frac{I_{u H}}{I_{u}}\right)^{2}=\int_{\eta_{c}}^{\infty} \frac{4}{70.78^{5 / 6} \eta^{5 / 3}} d \eta=\frac{6}{70.78^{5 / 6}} \eta_{c}{ }^{-2 / 3}$

Thus an approximate expression for $n_{c}$ is obtained:

$n_{c}=\eta_{c} \frac{U}{{ }^{{ }_{L} u}}=\frac{U}{{ }^{L_{u} u}}\left(\frac{6^{3 / 2}}{70.78^{5 / 4}} \frac{I_{u}^{3}}{I_{u H}^{3}}\right)=0.0716 \frac{U}{{ }^{L_{u u}}}\left(\frac{I_{u}}{I_{u H}}\right)^{3}$

Note that this relationship uses the full spectrum value of ${ }^{\mathrm{x}} L_{u}$ and $U$ is the mean velocity of atmospheric flow with full spectrum present.

\section{$\underline{2.5 \text { Wind Simulation }}$}

In a wind simulation the non-dimensional power spectrum based on mean velocity should be the same as at full scale for each sub interval. In a partial simulation this cannot be achieved over the whole spectrum but the aim is to achieve the match at high frequencies. This implies that for any particular subinterval at high frequencies:

$\frac{n_{m} S_{u m}}{U_{m}^{2}}=\frac{n_{p} S_{u p}}{\left(U_{p}+u_{L p}\right)^{2}}$

where subscripts $\mathrm{m}$ and $\mathrm{p}$ denote model scale and prototype (i.e. full-scale) quantities respectively. In this relationship the averaging time for the spectrum is over one sub-interval. The rapid equilibrium assumption implies that, just as with $I_{u H}$, at high frequencies the non-dimensional spectrum $S_{u H p}^{*}=n_{p} S_{u p} /\left(U_{p}+u_{L p}\right)^{2}$ is also a flow 
constant. In full scale field measurements the spectrum is evaluated over a large number of subintervals and is normalized by the mean velocity $U_{p}$. So, if we normalize using $U_{p}$ within each subinterval we would now have a variable non-dimensional spectrum $\frac{n_{p} S_{u p}}{U_{p}^{2}}=S_{u H p}^{*}\left(1+u_{L p} / U_{p}\right)^{2}$. However, averaging over many sub-intervals (denoted as before by a double over-bar), leads to:

$\frac{n_{p} \overline{\overline{S_{u p}}}}{U_{p}^{2}}=S_{u H p}^{*}\left(1+I_{u L p}^{2}\right) \approx S_{u H p}^{*}$

The approximation that $S_{u H p}^{*}\left(1+I_{u L p}^{2}\right) \approx S_{u H p}^{*}$ is good for typical values of $I_{u L p}$ in the range 0.1 to 0.2 . Therefore, Eq. (15a) may be expressed as:

$\frac{n_{m} S_{u m}}{U_{m}^{2}}=\frac{n_{p} \overline{\overline{S_{u p}}}}{U_{p}^{2}}$

This relationship implies that at high frequencies our modeled non-dimensional spectrum for a subinterval, should match the non-dimensional full spectrum averaged over the full sample time (e.g. one hour) at full scale.

At high frequencies the von Karman model of the power spectrum (Eq. 11) may be written as:

$\frac{n S_{u}}{U^{2}}=\frac{4 I_{u}^{2}}{70.78^{5 / 6}\left(\frac{n^{\times} L u}{U}\right)^{2 / 3}}$

Also the non-dimensional frequency, $n b / U$, where $b$ is a reference dimension, must match at model and prototype scale. This implies that:

$\frac{n_{m} b_{m}}{U_{m}}=\frac{n_{p} b_{p}}{U_{p}}$

Note that for a single sub-interval we would match model non-dimensional frequency to $n_{p} b_{p} /\left(U_{p}+u_{L p}\right)$, but when evaluating the full scale spectrum over the full sample time, as in Eq. (15c), the average value $\overline{\overline{n_{p} b_{p} /\left(U_{p}+u_{L p}\right)}}=n_{p} b_{p} / U_{p}$ is applicable.

Combining Eqs. (15c), (16) and (17) we find that $I_{u m} /\left(\frac{{ }^{x} L_{u m}}{b_{m}}\right)^{1 / 3}=I_{u p} /\left(\frac{{ }^{x} L_{u p}}{b_{p}}\right)^{1 / 3}$ which leads to the requirement that the ratio of model turbulence intensity to prototype turbulence intensity should be governed by:

$\frac{I_{u m}}{I_{u p}}=\left(\frac{{ }^{x} L_{u m}}{{ }^{x} L_{u p}}\right)^{1 / 3}\left(\frac{b_{p}}{b_{m}}\right)^{1 / 3}$

In a full turbulence simulation $\left(\frac{{ }^{\mathrm{x}} L_{u m}}{{ }^{\mathrm{x}} L_{u p}}\right)=\left(\frac{b_{p}}{b_{m}}\right)$ and it is required to have $I_{u m}=I_{u p}$. However, in a partial turbulence

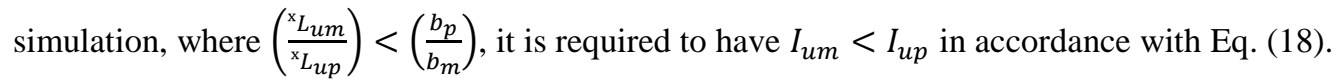


Equations 14 and 18 imply that at the dividing frequency, $n_{c}$, the ratio of the eddy wave length $l=U / n$ to the building dimension $b$ is approximately $14{ }^{\mathrm{x}} \mathrm{L}_{u m} / b_{m}$. This leads to the conclusion that if for example the requirement for validity of the quasi-steady assumption were to be set at $l / b>10$ then ${ }^{\mathrm{x}} L_{u m} / b_{m}$ must be greater than 0.7. In the present experiments, taking building height to be the relevant dimension, ${ }^{\mathrm{x}} L_{u m} / b_{m} \approx 0.4$ and 0.7 on the Silsoe cube and TTU building respectively (Section 3.1). Thus, if one accepts a criterion of $l / b>10$ as a requirement then the present experiments appear to be on the borderline of applicability of the quasi steady assumption and, as such, represent interesting test cases.

There are other integral scales similar to ${ }^{\mathrm{x}} L_{u}$ that are linked with the other turbulence velocity components, $v$ and $w$, in the lateral and vertical directions. Therefore, there are similar relationships for those components which should also be adhered to. However, typically it is found that if Eq. (18) is used to set the ratio $I_{u m} / I_{u p}$ then the equivalent ratios for the other turbulence velocity components fall into line fairly well.

\subsection{PTS method for determining the peak pressure coefficients}

In a partial turbulence simulation the sample period can be divided into subintervals of sufficient duration that peak values occurring in them may be treated as independent events. The peak pressure $\hat{p}$ in any one subinterval may be written as:

$\hat{p}=\frac{1}{2} \rho Q^{2} \hat{C}$

where $\hat{C}$ is the peak pressure coefficient that occurred during the subinterval. Strictly $\hat{C}$ is a function of flow angle (which would make it a function of low frequency lateral and vertical turbulence fluctuations) but as explained earlier we are deferring consideration of flow angle to a later section on 3DPTS. The resultant wind speed $Q$ for the subinterval is given by:

$Q^{2}=\left(U+u_{L}\right)^{2}+v_{L}^{2}+w_{L}^{2}$

where each of the low frequency turbulent velocity components $u_{L}, v_{L}, w_{L}$, may be regarded as constant during the subinterval. Therefore in each subinterval:

$\hat{p}=\frac{1}{2} \rho\left(\left(U+u_{L}\right)^{2}+v_{L}^{2}+w_{L}^{2}\right) \hat{C}$

In Eq. (21) the terms $v_{L}^{2}$ and $w_{L}^{2}$ are very small relative to $\left(U+u_{L}\right)^{2}$. Therefore, to simplify the analysis they will be ignored. Also, we define $\zeta \equiv \frac{u_{L}}{U}$. Then Eq. (21) may be used to obtain: 
$\hat{C}_{p}=\frac{\hat{p}}{\frac{1}{2} \rho U^{2}}=(1+\zeta)^{2} \hat{C}$

where $\hat{C}_{p}$ may be regarded as the peak pressure coefficient for a single subinterval based on the mean velocity of the full sample period with full spectrum turbulence present. The peak over all subintervals may be written:

$\hat{C}_{p M A X}=\left\langle(1+\zeta)^{2} \hat{C}\right\rangle_{M A X}$

where we have used the notation \langle\rangle$_{M A X}$ to denote the maximum value out of all the subintervals that make up the full sample period. For each subinterval there will be a combination of $\zeta$ and subinterval peak coefficient $\hat{C}$.

In the partial turbulence simulation we can estimate the probability that the peak pressure coefficient will not exceed a value $\hat{C}$ in a subinterval. This probability is in general described well by the Fisher Tippet Type I distribution.

$F(\hat{C})=\exp (-\exp (-a(\hat{C}-b)))$

where $a$ and $b$ are constants that can be determined experimentally. The probability that the pressure coefficient will exceed $\hat{C}$ in a subinterval is $G=1-F(\hat{C})$. From Eq. (22) we may replace $\hat{C}$ in Eq. (24) by $\frac{\hat{C}_{p}}{(1+\zeta)^{2}}$. Therefore, the probability that $\hat{C}_{p}$ will be exceeded for a given value of $\zeta$ is:

$G\left(\hat{C}_{p} / b, \zeta\right)=1-\exp \left(-\exp \left(-a b\left(\frac{\hat{C}_{p} / b}{(1+\zeta)^{2}}-1\right)\right)\right)$

For a given subinterval the probability of $\zeta$ being in the range $\zeta$ to $\zeta+\delta \zeta$ is $f_{\zeta}(\zeta) \delta \zeta$, in which $f_{\zeta}$ is the probability density of $\zeta$. Therefore, the probability of $\hat{C}_{p}$ being exceeded for all values of $\zeta$ is:

$G\left(\hat{C}_{p} / b\right)=\int_{-\infty}^{\infty} f_{\zeta}(\zeta)\left[1-\exp \left(-\exp \left(-a b\left(\frac{\hat{C}_{p} / b}{(1+\zeta)^{2}}-1\right)\right)\right)\right] d \zeta$

The probability distribution of wind turbulence in a generic boundary layer (i.e. one free from local aerodynamic effects of upwind structures) is generally Gaussian or near Gaussian (Chu et al, 1996; Lu and Porte-Agel, 2010). So it is assumed for the probability density of the large scale turbulence that:

$f_{\zeta}(\zeta)=\frac{1}{\sqrt{2 \pi} I u L} e^{-\frac{1}{2}\left(\frac{\zeta}{I_{u L}}\right)^{2}}$

Therefore, we deduce that the probability of $\hat{C}_{p}$ being exceeded for all values of $\zeta$ is:

$G\left(\hat{C}_{p} / b\right)=\int_{-\infty}^{\infty} \frac{1}{\sqrt{2 \pi} I_{u L}} e^{-\frac{1}{2}\left(\frac{\zeta}{I_{u L}}\right)^{2}}\left[1-\exp \left(-\exp \left(-a b\left(\frac{\hat{C}_{p} / b}{(1+\zeta)^{2}}-1\right)\right)\right)\right] d \zeta$

Note that if we define:

$\xi \equiv \frac{\zeta}{I_{u L}}$ 
Then Eq. (28) can be written as:

$G\left(\hat{C}_{p} / b\right)=\int_{-\infty}^{\infty} \frac{1}{\sqrt{2 \pi}} e^{-\frac{1}{2} \xi^{2}}\left[1-\exp \left(-\exp \left(-a b\left(\frac{\hat{C}_{p} / b}{\left(1+I_{u L} \xi\right)^{2}}-1\right)\right)\right)\right] d \xi$

The parameters $a$ and $b$ can be measured from time histories of pressures in partial simulations. Then, given the turbulence intensity of the low frequency fluctuations, $I_{u L}$, the probability of exceeding a given value of $\hat{C}_{p} / b$ in a single subinterval can be computed by numerical integration using Eq. (30). If our full sample period is one hour of wind in which we have $N$ subintervals then the highest pressure coefficient will have probability $1 / N$ of being exceeded. For example, if our subintervals on the model scale up to be 1.5 seconds at full scale then the highest value of $\hat{C}_{p} / b$ will have $1 / N=1.5 / 3600=0.00042$ probability of being exceeded in one hour and this will represent the mode of the peak values.

It can be noted that the probability of the mode value not being exceeded during one hour is $P_{N}=\left(1-\frac{1}{N}\right)^{N}$. As $N$ increases above about 10 this rapidly asymptotes to $P_{N}=0.3679$. So the mode of the distribution has about $37 \%$ probability of not being exceeded in the hour of wind. If we want to set the probability of non-exceedance in $N$ subintervals to some other target value, $P_{\text {tar }}$ (a value of 0.78 is often used in wind tunnel studies), then this is equivalent to setting the probability per subinterval $P$ such that $P^{N}=P_{t a r}$. The probability of exceedance per subinterval at which to evaluate $\hat{C}_{p} / b$ is then:

$G=1-P_{\operatorname{tar}^{1 / N}}$

If, for example $P_{t a r}=0.78$, and $N=2400$ then $G=0.00010$.

It can be shown that the mean, or expected value, of the Fisher Tippet Type I distribution is at probability:

$P_{\text {tar }}=e^{-e^{-\gamma}}=e^{-e^{-0.5772}}=0.5704$

where $\gamma$ is Euler's constant.

When predicting the most probable peak in a single hour (or some other selected full sample period) of wind the relationships for the mode would be the most applicable. When comparing with the average of many events such as the records for the Silsoe Cube and TTU building in this paper, the mean or expected peak prediction is the most applicable. 
Figure 2 illustrates the results of the numerical integration of Eq. (30) for various values of $a b$ in the typical range with low frequency turbulence intensity $I_{u L}=0.2$. It can be seen that the peak value of $\hat{C}_{p} / b$ corresponding to the selected target probability of 0.00042 depends on the value of $a b$.

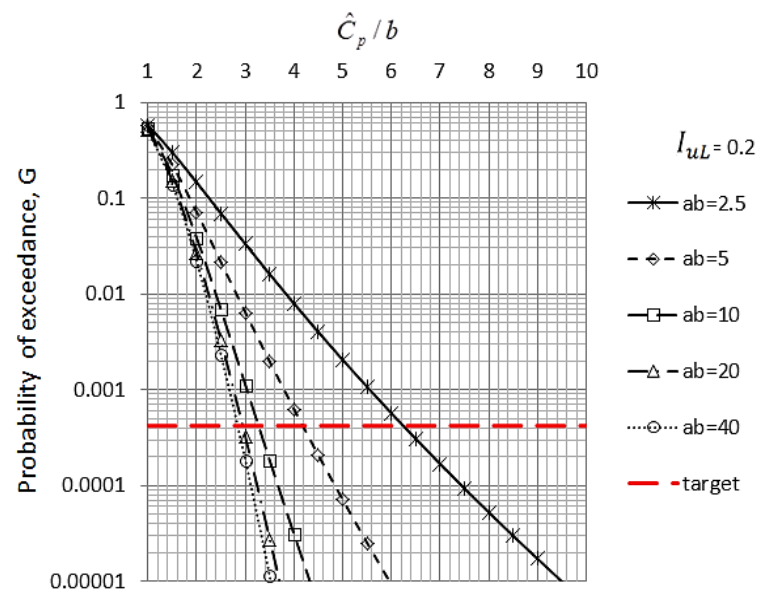

Fig.2. Probability of exceeding $\hat{C}_{p} / b$ for $I_{u L}=0.2$ and various $a b$ values, from Eq. (30)

The peak pressure coefficients calculated using the procedure described above are based on mean hourly dynamic pressure. The pressure coefficient $\hat{C}_{p, 3 \text { sec }}$ based on 3-second gust dynamic pressure, for example, can be obtained by re-scaling using:

$\hat{C}_{p, 3 \sec }=\hat{C}_{p}\left(\frac{U}{\hat{U}_{3 \sec }}\right)^{2}$

The above analysis has been in the context of pressure coefficient. However, the same procedure can be applied to any other quantity such as a force or moment coefficient, just so long as the behavior of the pressure, force or moment that is involved varies as velocity squared. In principle other probability density functions than Gaussian for the low frequency turbulence could be used for non-generic boundary layer situations if data are available.

It is worth noting that in a partial turbulence simulation it is not necessary to sample for the full number $N$ of subintervals in one hour (at full scale) because without the low frequency component of turbulence, statistical stability of the parameters $a$ and $b$ can be obtained with fewer. At full scale, with the full turbulence spectrum present, it is widely recognized that $T_{s}=1$ hour is sufficient sample time to achieve stable statistics when measuring fluctuating wind loads. In fact full scale sample times as short as 10 minutes are sometimes used. Here we assume 1 hour at full scale is the target. The representative characteristic time for the turbulence is $\frac{{ }^{x} L_{u}}{U}$. With the full spectrum present a 
representative range for the characteristic time of many full scale measurements is 5 to 10 seconds, meaning that in one hour there are between 360 and 720 characteristic times, and this has been found to give reasonable statistical stability. Therefore, if in a partial simulation the total sample time is long enough to represent this range of characteristic times, bearing in mind the integral scale and speed of the tests, then statistical stability can also be expected to be achieved. Typical values of ${ }^{\mathrm{x}} L_{u}$ in partial turbulence simulations are of order $0.5 \mathrm{~m}$ and typical velocities for measurements are say $20 \mathrm{~m} / \mathrm{s}$, implying a characteristic time on the order of $0.025 \mathrm{~s}$. To be in the range of 360 to 720 characteristic times the total sample time in the tests therefore needs to be at minimum 9 to 18 seconds. This is not very demanding and much longer sample times than these minimum values were used in the current work.

\subsection{DPTS Method for determining the peak pressure coefficients}

In the previous section the presence of low frequency lateral and vertical turbulence fluctuations were ignored. The real situation is more complex due to low frequency turbulence causing variations not only in longitudinal velocity but also in the flow azimuthal angle $\varphi$ and pitch angle $\theta$ from subinterval to subinterval. The behavior of peak pressures will tend to be different for different flow angles. To allow for this the peak pressure $\hat{p}$ in any one subinterval is now written as:

$\hat{p}=\frac{1}{2} \rho Q^{2} \hat{C}(\varphi, \theta)$

where $\hat{C}(\varphi, \theta)$ is the peak pressure coefficient that occurred during the subinterval and the angles $\varphi, \theta$ may be regarded as a constant within the subinterval. The resultant wind speed $Q$ for the subinterval is still given by:

$Q^{2}=\left(U+u_{L}\right)^{2}+v_{L}^{2}+w_{L}^{2}$

where each of the low frequency turbulent velocity components $u_{L}, v_{L}, w_{L}$, like the flow angles, may be regarded as constant during the subinterval. Therefore in each subinterval:

$\hat{p}=\frac{1}{2} \rho\left[U^{2}+2 U u_{L}+q_{L}^{2}\right] \hat{C}(\varphi, \theta)$

where $q^{2}=u_{L}^{2}+v_{L}^{2}+w_{L}^{2}$. The last term in Eq. (40) is ignored as being a couple of orders of magnitude below the

first. We also note that for practical ranges of turbulence intensity $\varphi=\frac{v_{L}}{U}$ and $\theta=\frac{w_{L}}{U}$, and we define $\eta=\frac{u_{L}}{U}$. Then

Eq. (40) may be simplified and expressed as:

$\frac{\hat{p}}{\frac{1}{2} \rho U^{2}}=(1+2 \eta) \hat{C}(\varphi, \theta)$ 
Equation (41) may be regarded as the expression for the peak pressure coefficient for a single subinterval based on the mean velocity over the full sample period. The peak over all subintervals may be written as:

$\hat{C}_{p}=\langle(1+2 \eta) \hat{C}(\varphi, \theta)\rangle_{M A X}$

where we have again used the notation \langle\rangle$_{M A X}$ to denote the maximum value out of all the subintervals that make up the full sample period. For each subinterval there will be a combination of $\eta, \varphi, \theta$ and also some random variations in the value of subinterval peak coefficient $\hat{C}$. To proceed any further we need to adopt a probabilistic methodology. In the partial turbulence simulation we can set up the angles $\varphi, \theta$ and measure the probability that the peak pressure coefficient will not exceed a value $\hat{C}$ in a subinterval. The Fisher Tippet Type I distribution is now reexpressed to allow dependence on $\varphi$ and $\theta$.

$F_{\varphi, \theta}(\hat{C}, \varphi, \theta)=\exp \left(-\exp \left(-a_{\varphi \theta}\left(\hat{C}-b_{\varphi \theta}\right)\right)\right)$

where $a_{\varphi \theta}$ and $b_{\varphi \theta}$ are constants that can be determined experimentally as functions of the flow angles $\varphi, \theta$. From the probability of $\hat{C}$ given in Eq. 43 the probability density of $\hat{C}$ is:

$f_{c}(\hat{C}, \varphi, \theta)=a_{\varphi \theta} e^{-a_{\varphi \theta}\left(\hat{C}-b_{\varphi \theta}\right)} e^{-e^{-a_{\varphi \theta}\left(\widehat{C}-b_{\varphi \theta}\right)}}$

For a given subinterval the probability of $\hat{C}$ being in the range $\hat{C}$ to $\hat{C}+\delta \hat{C}$ is $f_{c} \delta \hat{C}$. The probability that the angle $\varphi$ is in the range $\varphi$ to $\varphi+\delta \varphi$ is $f_{\varphi}(\varphi) \delta \varphi$ and the probability that the angle $\theta$ is in the range $\theta$ to $\theta+\delta \theta$ is $f_{\theta}(\theta) \delta \theta$, where $f_{\varphi}$ and $f_{\theta}$ are probability densities of $\varphi$ and $\theta$ respectively. Therefore, the probability of all three occurring is $f_{c}(\hat{C}, \varphi, \theta) f_{\varphi}(\varphi) f_{\theta}(\theta) \delta \hat{C} \delta \varphi \delta \theta$. In boundary layer flow there is correlation between $\eta$ and $\theta$. Therefore, if we want the probability of $\eta$ also being in the range $\eta$ to $\eta+\delta \eta$ it must be expressed as $f_{c}(\hat{C}, \varphi, \theta) f_{\varphi}(\varphi) f_{\eta \theta}(\eta, \theta) \delta \hat{C} \delta \eta \delta \varphi \delta \theta$ where $f_{\eta \theta}$ is the joint probability density of $\eta$ and $\theta$. We want the probability $G\left(\hat{C}_{p}\right)$, of $\hat{C}_{p}$ in Eq. (42) exceeding a certain value. We could initially ask for the probability of $\hat{C}_{p}$ being exceeded while $\eta$ is at a certain value. This can be obtained by integrating over all values of $\varphi$ and $\theta$, and from $\hat{C}=\frac{\hat{C}_{p}}{(1+2 \eta)}$ to infinity:

$G\left(\hat{C}_{p}, \eta\right)=\int_{\frac{\hat{C}_{p}}{(1+2 \eta)}}^{\infty} \int_{-\pi}^{+\pi} \int_{-\pi}^{+\pi} f_{c}(\hat{C}, \varphi, \theta) f_{\varphi}(\varphi) f_{\eta \theta}(\eta, \theta) d \varphi d \theta d \hat{C}$

Then we need to do the integration over all $\eta$ to obtain the total probability of exceeding $\hat{C}_{p}$ :

$G\left(\hat{C}_{p}\right)=\int_{-\infty}^{\infty} \int_{\frac{\hat{C}_{p}}{(1+2 \eta)}}^{\infty} \int_{-\pi}^{+\pi} \int_{-\pi}^{+\pi} f_{c}(\hat{C}, \varphi, \theta) f_{\varphi}(\varphi) f_{\eta \theta}(\eta, \theta) d \varphi d \theta d \hat{C} d \eta$

The integration with respect to $\varphi$ can be done first: 
$G\left(\hat{C}_{p}\right)=\int_{-\infty}^{\infty} \int_{\frac{\widehat{C}_{p}}{(1+2 \eta)}}^{\infty} \int_{-\pi}^{+\pi}\left(\int_{-\pi}^{+\pi} f_{c}(\hat{C}, \varphi, \theta) f_{\varphi}(\varphi) d \varphi\right) f_{\eta \theta}(\eta, \theta) d \theta d \hat{C} d \eta$

Calling this inner integral $I_{1}$, we have:

$I_{1}(\hat{C}, \theta)=\int_{-\pi}^{+\pi} f_{c}(\hat{C}, \varphi, \theta) f_{\varphi}(\varphi) d \varphi=\int_{-\pi}^{+\pi} a_{\varphi \theta} e^{-a_{\varphi \theta}\left(\hat{C}-b_{\varphi \theta}\right)} e^{-e^{-a_{\varphi \theta}\left(\hat{C}-b_{\varphi \theta}\right)}} \frac{1}{\sqrt{2 \pi} \sigma_{\varphi}} e^{-\frac{1}{2}\left(\frac{\varphi^{2}}{\sigma_{\varphi}^{2}}\right)} d \varphi$

$=\frac{1}{\sqrt{2 \pi} \sigma_{\varphi}} \int_{-\pi}^{+\pi} a_{\varphi \theta} e^{-\left(a_{\varphi \theta}\left(\hat{C}-b_{\varphi \theta}\right)+e^{-a_{\varphi \theta}\left(\widehat{C}-b_{\varphi \theta}\right)}+\frac{1}{2}\left(\frac{\varphi^{2}}{\sigma_{\varphi}^{2}}\right)\right)} d \varphi$

This is best evaluated numerically and it needs to be done for a range of values of $\hat{C}$ and $\theta$. Equation (47) then becomes:

$G\left(\hat{C}_{p}\right)=\int_{-\infty}^{\infty} \int_{\frac{\hat{C}_{p}}{(1+2 \eta)}}^{\infty} \int_{-\pi}^{+\pi} I_{1}(\hat{C}, \theta) f_{\eta \theta}(\eta, \theta) d \theta d \hat{C} d \eta$

To evaluate this integral we need the joint probability density $f_{\eta \theta}(\eta, \theta)$. The probabilities of $\eta$ and $\theta$ tend to follow a Gaussian form but are correlated. Using the Gaussian form for two correlated variables, with correlation coefficient $r$, the joint probability density is expressed as:

$f_{\eta \theta}(\eta, \theta)=\frac{1}{2 \pi \sigma_{\eta} \sigma_{\theta}\left(1-r^{2}\right)^{1 / 2}} \exp \left[-\frac{1}{2\left(1-r^{2}\right)}\left(\frac{\eta^{2}}{\sigma_{\eta}^{2}}-\frac{2 r \eta \theta}{\sigma_{\eta} \sigma_{\theta}}+\frac{\theta^{2}}{\sigma_{\theta}^{2}}\right)\right]$

Therefore our next step is to evaluate:

$I_{2}(\hat{C}, \eta)=\int_{-\pi}^{+\pi} I_{1}(\hat{C}, \theta) f_{\eta \theta}(\eta, \theta) d \theta=\frac{1}{2 \pi \sigma_{\eta} \sigma_{\theta}\left(1-r^{2}\right)^{1 / 2}} \int_{-\pi}^{+\pi} I_{1}(\hat{C}, \theta) \exp \left[-\frac{1}{2\left(1-r^{2}\right)}\left(\frac{\eta^{2}}{\sigma_{\eta}^{2}}-\frac{2 r \eta \theta}{\sigma_{\eta} \sigma_{\theta}}+\frac{\theta^{2}}{\sigma_{\theta}^{2}}\right)\right] d \theta$

Again, the integration is best done numerically. The next step after obtaining the function $I_{2}(\hat{C}, \eta)$ is to evaluate:

$G\left(\hat{C}_{p}\right)=\int_{-\infty}^{\infty} \int_{\frac{\widehat{C}_{p}}{(1+2 \eta)}}^{\infty} I_{2}(\hat{C}, \eta) d \hat{C} d \eta$

This is achieved by first evaluating:

$I_{3}\left(\hat{C}_{p}, \eta\right)=\int_{\frac{\widehat{C}_{p}}{(1+2 \eta)}}^{\infty} I_{2}(\hat{C}, \eta) d \hat{C}$

where $\hat{C}_{p}$ is a selected value of peak pressure coefficient. The integration is again done numerically. Then the final step is to evaluate:

$G\left(\hat{C}_{p}\right)=\int_{-\infty}^{\infty} I_{3}(\eta) d \eta$ 
Equation (54) gives us the probability that a given value of $\hat{C}_{p}$ will be exceeded in a particular subinterval in the full turbulence. The computations need to be repeated for a range of values of $\hat{C}_{p}$. To summarize, the various steps of the procedure are:

- $\quad a$ and $b$ are determined as a function of $\phi$ and $\theta$ from tests which measure $\hat{C}$ for each test subinterval, using the usual ranking method of fitting to extremes.

- Step 1 is repeated for a number of azimuth $\varphi$ and pitch $\theta$ angles (In this paper 11 azimuth angles at 3 degree increments and 6 pitch angles at 2 degree increments were tested).

- $\quad I_{1}$ is evaluated using numerical integration over $\varphi$ for each value of $\theta$ and a selected range of values of peak pressure coefficient $\hat{C}$.

- $\quad I_{2}$ is evaluated using numerical integration over $\theta$ for each of value of $\eta$ and $\hat{C}$. In the present work a value of $r=-0.3$ was used based on typical values of $\sigma_{u} / u_{\tau} \approx 2.5$ and $\sigma_{w} / \sigma_{u} \approx 0.55$ for the full spectrum near the ground, where $u_{\tau}=\sqrt{-\overline{u w}}$, (ESDU, 2001). Since the bulk of the turbulence energy in the full spectrum was in the low frequencies in the present studies it was assumed that the value for $r$ for the full spectrum would also apply to the low frequencies.

- $\quad I_{3}$ is evaluated using numerical integration over $\hat{C}$ for each value of $\eta$ and $\hat{C}_{p}$.

- $G\left(\hat{C}_{p}\right)$ is evaluated using numerical integration over $\eta$ for each of the selected values of $\hat{C}_{p}$.

- From the graph of $G\left(\hat{C}_{p}\right)$ versus $\hat{C}_{p}$, the expected value of peak pressure coefficient for the full turbulence sample period is interpolated.

Note that in evaluating the $I_{1}(\hat{C}, \theta)$ in Eq. (48) and $I_{2}(\hat{C}, \eta)$ in Eq. (51), the limits of the integral are $-\pi: \pi$, theoretically. However, when doing experiments the variations in $\varphi$ and $\theta$ do not need to be this wide since typically very little contribution to the integral arises for angles greater than about 30 degrees.

\section{Experiments}

\subsection{Test building}

In order to check the efficacy of the PTS and 3DPTS methods described above the full-scale pressure coefficient data obtained by Richards and Hoxey (2012) on the Silsoe cube (a 6 m cube at full scale) and by Levitan and Mehta (1992a,b) on the Texas Tech University (TTU) building (a rectangular building with plan dimension of $9.144 m \times$ 
$13.72 \mathrm{~m}$ and $3.96 \mathrm{~m}$ height at full scale) were used as benchmarks for comparison. The Silsoe cube model was at 1:5 scale and TTU building model was at 1:6 scale. These are large scales but the structures themselves are relatively small when compared to most low rise buildings. Figure 3 shows the building models in the Wall of Wind (WOW) facility at Florida International University.

(a)

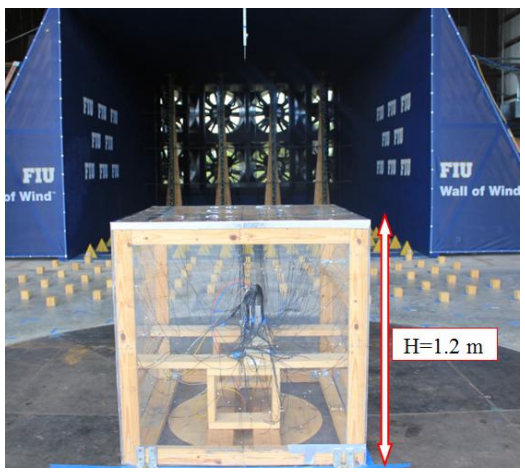

(b)

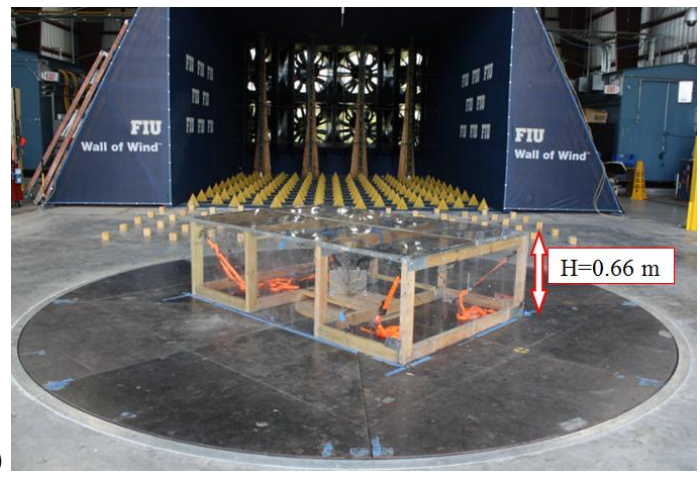

Fig.3. Building models tested in WOW: (a) Silsoe cube, (b) TTU building

As explained previously, the 3DPTS method requires a number of tests at different azimuth and pitch angles at small angle increments around the main wind direction under study. For the results presented for the 3DPTS method, one main wind direction tested was $45^{\circ}$. Based on past studies, this wind direction was selected as representative for generating high uplifts under conical vortices on flat rectangular roofs (Holmes, 2007). The range of azimuth angles was 15 degrees on either side of the desired wind direction in 3 degree intervals. This means that tests were needed to be performed from 30 to 60 degree wind directions in 3 degrees intervals resulting in 11 azimuth angles. In addition, models were pitched from -6 to +6 degrees in 2 degree intervals (a total of 7 pitch angles) (Fig. 4). To do so, a platform was built for the building models. Figure 5 shows the building models on the platform. For 3DPTS method, pressure results are given just for the taps on the roof. The pressure coefficients on the roof of each model were measured and compared for the cases of building model with and without the platform to make sure that the platform did not have any significant effect on the pressure results on the roof. The differences were very small. 

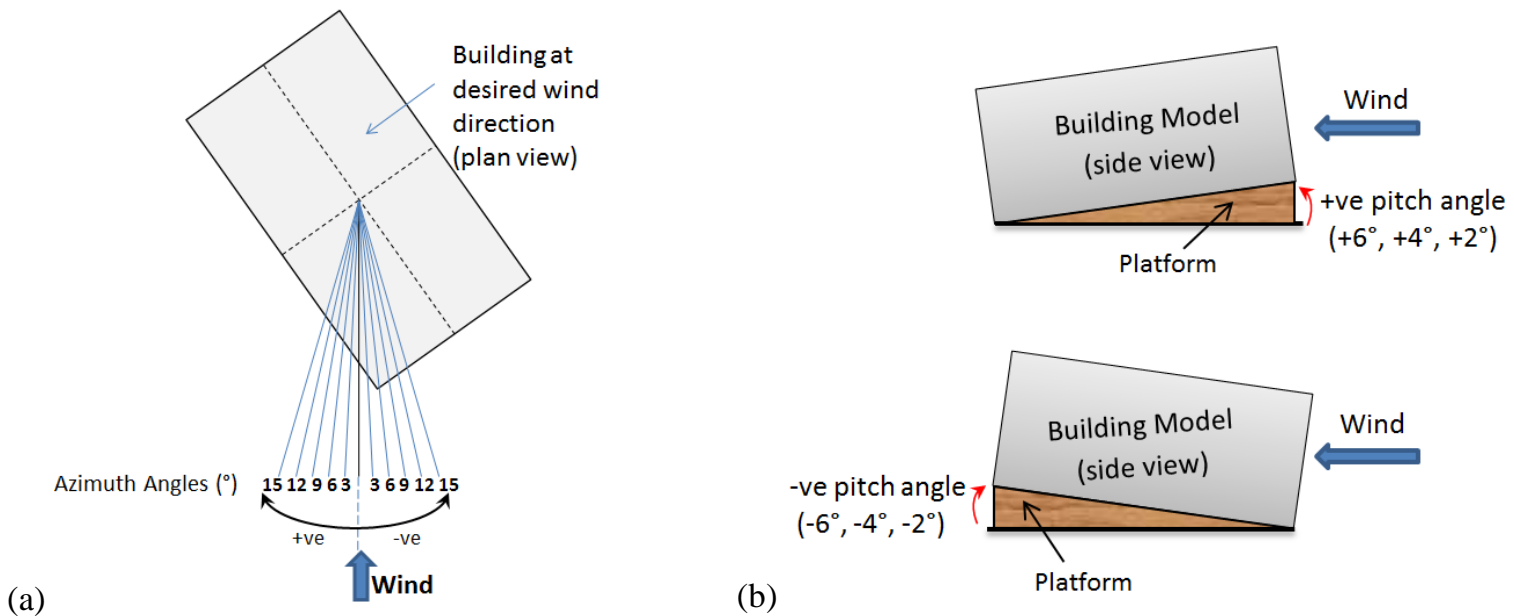

(b)

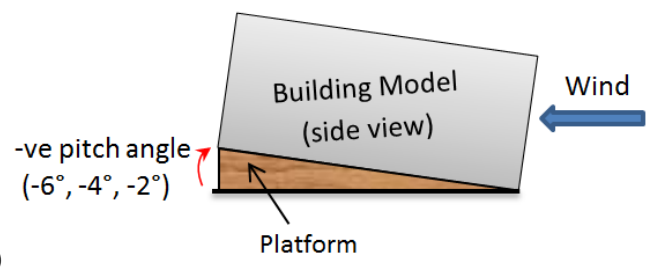

Fig.4. Definition of wind azimuth (a) and pitch angle (b)

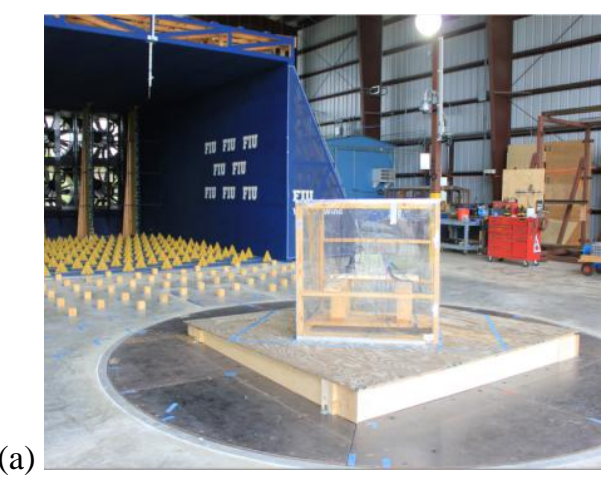

(b)

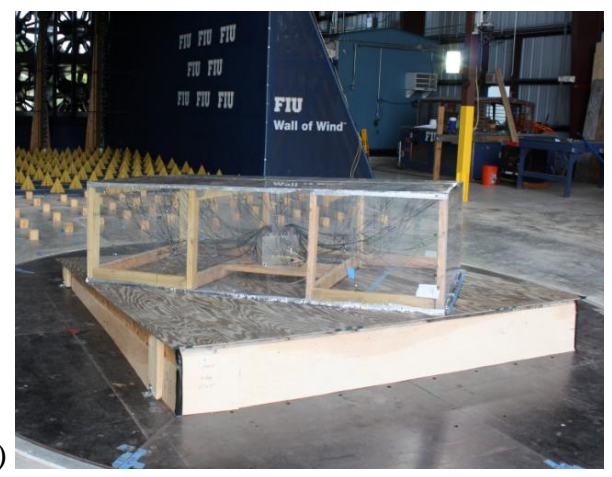

Fig.5. Building models tested in WOW for 3DPTS method: (a) Silsoe cube, (b) TTU building

Figure 6 shows the pressure tap locations, which matched the full-scale tap locations. It should be noted that the tap layout selected for the TTU tests is based on a newer tap configuration since the procedure reported by Levitan and Mehta (1992a). The new tap layout and raw experimental data was provided by Texas Tech University. However, to make comparisons possible with the available data in literature, locations of three critical taps named 50101, 50501, and 50901 that have been the subject of many simulations are also shown in Figure 6.b.” 
(a)

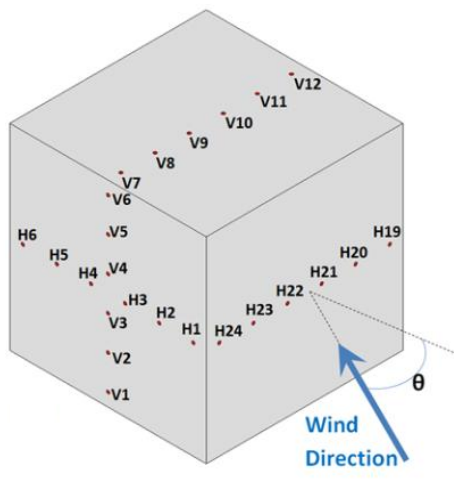

Fig.6. (a) Tap locations on Silsoe model, (b) Tap locations on TTU model roof

A 512 channel Scanivalve Corporation pressure scanning system was used for pressure measurements. Pressure data were acquired at a sampling frequency of $512 \mathrm{~Hz}$ for a period of 120 seconds (this more than satisfies the minimum requirements for sample time, discussed in Section 2.5, to obtain statistical stability when using the partial turbulence simulation approach). A transfer function designed for the tubing (Irwin et al, 1979) was used to correct for tubing effects. All pressure readings were low pass filtered at frequencies corresponding to the "low pass filter" frequency at full scale (see Tables 1 and 2).

\subsection{Wind flow}

Testing was performed in the 12-fan WOW open jet facility at FIU (Fig. 7a). This open jet facility can generate a $6.1 \mathrm{~m}$ wide and $4.3 \mathrm{~m}$ high wind field and speeds as high as $70 \mathrm{~m} / \mathrm{s}$. A set of triangular spires and floor roughness elements (Fig.7b) was used to generate the turbulence and boundary layer characteristics.
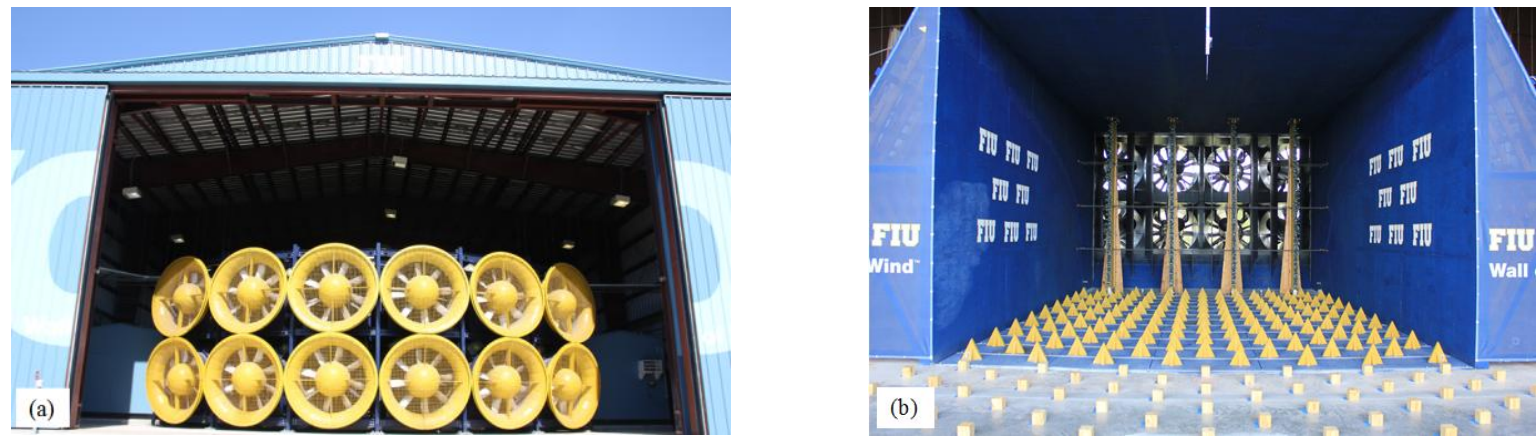

Fig.7. (a) Wall of Wind, Florida International University, (b) Spires and floor roughness elements

Figure 8 shows the comparison of the non-dimensional longitudinal turbulence power spectra for the full scale Silsoe cube, the Von Karman full spectrum of ABL flow in open terrain obtained from ESDU (2001) using two 
different $z_{0}$ values, and the WOW measured flow at the level of the top of the model in open terrain. The spectra are plotted in the non-dimensional form of $n S(n) / U^{2}$ versus $n z / U$, as suggested by Irwin (1998) and Richards et al (2007), where $z$ is a reference length taken here as the height of the Silsoe cube. The WOW spectrum matched the atmospheric boundary layer ESDU spectrum for a roughness length of about $z_{0}=0.1$ which is a somewhat higher roughness length than at the Silsoe site, but still within the range of $z_{0}$ considered as open terrain by ASCE (2010). Although the matching of spectra at high frequencies was not perfect it was felt worth proceeding with the PTS procedure to see how it performed. It is worth noting that when the spectrum at very high frequencies is too high one side effect is that a somewhat improved match is obtained at lower frequencies. Precisely how best to match the spectrum to obtain optimal results from the PTS approach is an interesting topic for future research that is being pursued further at the Wall of Wind facility.

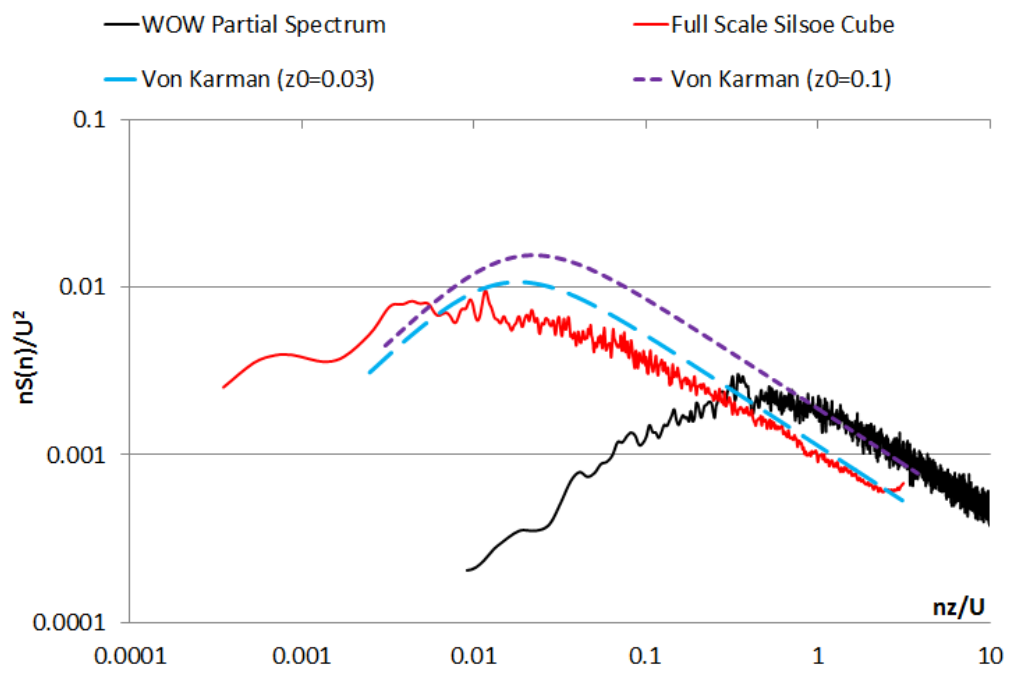

Fig.8. Comparison between full spectrum of ABL flow at Silsoe site and Von Karman full spectrum of ABL flow in open terrain with WOW partial spectrum in open terrain

\subsection{Wall of Wind scaling parameters}

In this section, a summary of the step by step procedure is presented for calculating the WOW scaling parameters used for determining the required probability level at which peak pressures were estimated:

- $\quad$ The missing low frequency turbulence intensity $I_{u L}$ was calculated using Eq. (9).

- The full scale gust, obtained by incorporating the effect of the missing low frequency turbulence, was estimated using the peak factor of 3.4 used in ASCE/SEI 7-10 (2010) for background turbulence using: 
$\widehat{U}_{L p}=U_{p}\left(1+3.4 I_{u L}\right)$

in which $U_{p}$ is the mean wind speed at full scale. Note that the peak factor for the low frequency gusts could alternatively have been calculated using ESDU (2001) based on the gust duration given by Equation 58 below and a slightly lower peak factor obtained. However, the end effect of the peak factor is to simply change the time scaling and the predicted peak pressure coefficients are relatively insensitive to minor differences in time scale.

- The speed scaling from model to full scale depends on what full scale (low frequency) speed fluctuation we assume we are modeling during a particular subinterval on the model. Since the low frequency fluctuations of full scale speed vary from subinterval to subinterval (Fig.1) there is a question as how to fix the speed scaling,. The most probable situation causing the highest wind load or local pressure is where a particularly high large scale gust blows through. Therefore, by far the largest contribution to probability of exceedance comes when the low frequency gust fluctuations are at high positive values. With this in mind the speed scaling for the present study was set such that the mean speed of the PTS tests corresponded to the low frequency gust speed calculated above in Eq. (55). Hence:

$\lambda_{U}=\frac{U_{m}}{\widehat{U}_{L p}}$

- From the length scale $\lambda_{L}=\frac{b_{m}}{b_{p}}$, and speed scale the time scale is then calculated as:

$\lambda_{t}=\lambda_{L} / \lambda_{U}$

- The equivalent gust-duration at full scale can be calculated using Eq. (58). Derivation of this equation is explained in more detail in Appendix A based on relationships for the effective filter frequency for a $\Delta t$ second moving average:

$t_{\text {Gust }}=0.45 / n_{c}$

This implies that in the Wall of Wind (WOW) the sample period could be regarded as being made up of a sequence of $t_{\text {Gust }}$ second duration gusts. Therefore, in dividing the sample period into subintervals the length of each subinterval needed to be in excess of $t_{\text {Gust }}$ seconds to avoid excessive correlation between events in adjacent subintervals. In other words, for $N_{\text {test }}$ subintervals, the length of each subinterval in partial simulation is $t_{\text {subinterval,PS }}=\frac{\text { test duration }}{N_{\text {test }}}$. The equivalent gust-duration at full scale is then 
$t_{\text {subinterval,FS }}=t_{\text {subinterval,PS }} / \lambda_{t}$ which should be in excess of $t_{\text {Gust }}$ seconds. Then, the required $G$ at which pressure coefficients are calculated can be obtained using:

$G=\frac{t_{\text {subinterval,FS }}}{\text { Full Scale time }(\text { e.g.1 hour })}$

- Finally, the full-scale equivalent peak pressure coefficient is obtained from intersecting the graph of $G\left(\hat{C}_{p}\right)$ versus $\hat{C}_{p}$ at $G$.

\section{Results and Discussions}

\subsection{Silsoe cube pressure results}

The pressure coefficient comparisons shown in this section are based on full-scale Silsoe measurement results given in Richards and Hoxey (2012) in which the pressure coefficients are defined in terms of the mean dynamic pressure:

$C_{\text {mean }}=\frac{P_{\text {mean }}}{q_{\text {mean }}}$

$C_{\text {peak }}=\frac{P_{\text {peak }}}{\left(3 q_{\text {mean }}\right)}$

where $P_{\text {mean }}$ is the mean pressure, $P_{\text {peak }}$ is the highest positive or lowest negative pressure observed during the test duration at the Silsoe site and $q_{\text {mean }}$ is the mean dynamic pressure defined as $0.5 \rho U^{2}$ ( $\rho=$ air density). The WOW mean and peak pressure data were obtained using the methods described in Sec. 2.6 and 2.7 for the PTS and 3DPTS methods respectively and were normalized in the same way as in Eqs. (60) and (61). Table 1 shows the test conditions for the Silsoe model in the WOW and at full-scale. The achieved power law coefficient for the mean velocity profile was 0.185 in the WOW tests.

Table 1. Test conditions for Silsoe cube model in the WOW and at Full-scale

\begin{tabular}{lll}
\hline Test Characteristics & Full Scale & Model scale $\left(\boldsymbol{\lambda}_{\mathbf{L}}=\frac{\mathbf{1}}{\mathbf{5}}\right)$ \\
\hline & $I_{u}=0.1955$ & $I_{u H}=0.074$ \\
Turbulence intensity & $I_{v}=0.15$ & $I_{v H}=0.073$ \\
& $I_{w}=0.078$ & $I_{w H}=0.063$ \\
Integral length scale & $L_{u}^{x}=53 \mathrm{~m}$ & $L_{u}^{x}=0.46 \mathrm{~m}$ \\
Reference height & $Z_{r e f}=6 \mathrm{~m}$ & $Z_{r e f}=1.2 \mathrm{~m}$ \\
Mean wind speed & $U_{F S}=9.52 \mathrm{~m} / \mathrm{s}$ & $U_{P S}=21.05 \mathrm{~m} / \mathrm{s}$ \\
Test duration & $T_{F S}=12 \mathrm{~min}$ & $T_{P S}=2 \mathrm{~min}$ \\
Low pass filter frequency & $8 \mathrm{~Hz}$ & $55 \mathrm{~Hz}$ \\
\hline
\end{tabular}


Expected peak pressure coefficients were obtained with the WOW sample time divided into 100 subintervals. This means that each subinterval in model scale was $120 / 100=1.2$ sec. Based on the time scale, each subinterval was equivalent at full scale to $1.2 / 0.145=8.28 \mathrm{sec}$. So, the targeted probability to obtain the mode of the peak pressure coefficient for 12 min duration in full spectrum conditions was $G=8.28 /(12 \times 60)=0.011$. To obtain the expected peak, which is peak reported in subsequent discussion, a slightly lower probability given by $G=1-$ $0.57^{0.011}=0.0062$ (Eq. (31)) was used in the analysis.

Figure 9 shows comparisons of wall pressure coefficients obtained in the WOW using the PTS method and at full scale (see Fig. 6.a for the pressure tap notation). For the peak coefficients the observed peak values without any PTS correction are also shown. The results show that after the PTS correction generally good agreement with full scale for both the mean and peak coefficients is obtained. The peak values are brought much closer to the full scale results when compared with the raw observed peaks. It should be noted that taps H19 to H24 undergo a transition from reattached to separated flow regions which can be seen from the transitioning of the pressure coefficients from positive to negative values as the wind direction increases from zero to 90 degrees. It can be seen the PTS method continues to predict the full scale results with reasonable accuracy as the flow transitions from the attached to detached condition. Figure 10 shows the overall minimum and maximum of peak pressure coefficients on the walls considering all directions. The agreement is within $10 \%$ except for the negative peak on tap H20 which had the lowest magnitude value. With respect to overall tendencies, the peak negative PTS values in Figure 10 are mostly slightly higher than at full scale, whereas the peak positive values are mostly slightly lower.
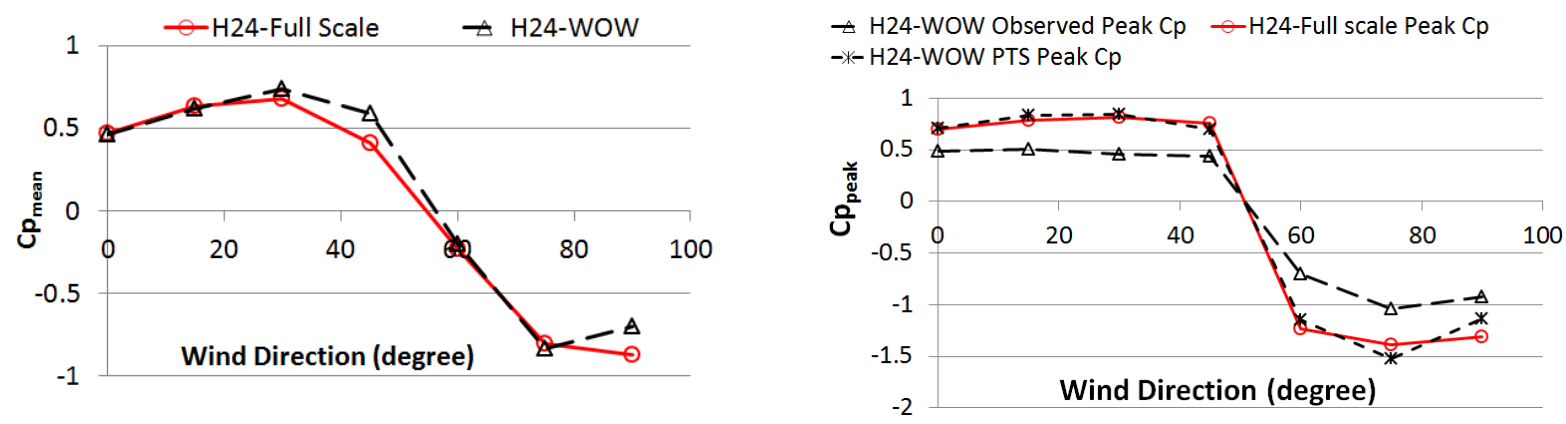

Fig.9. Silsoe cube wall $\mathrm{Cp}$ values comparisons vs. wind direction 

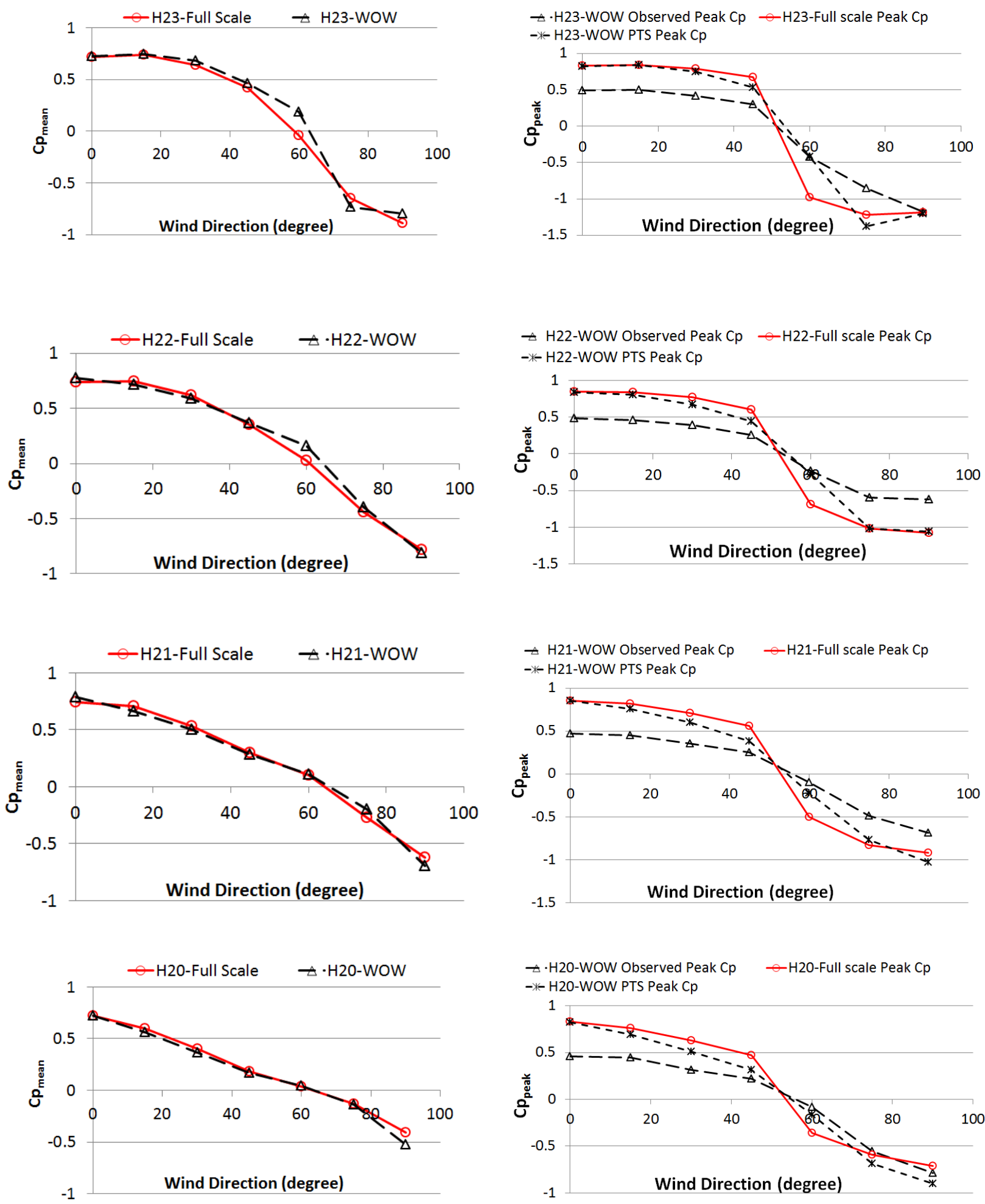

Fig.9. (Cont.). Silsoe cube wall Cp values comparisons vs. wind direction 

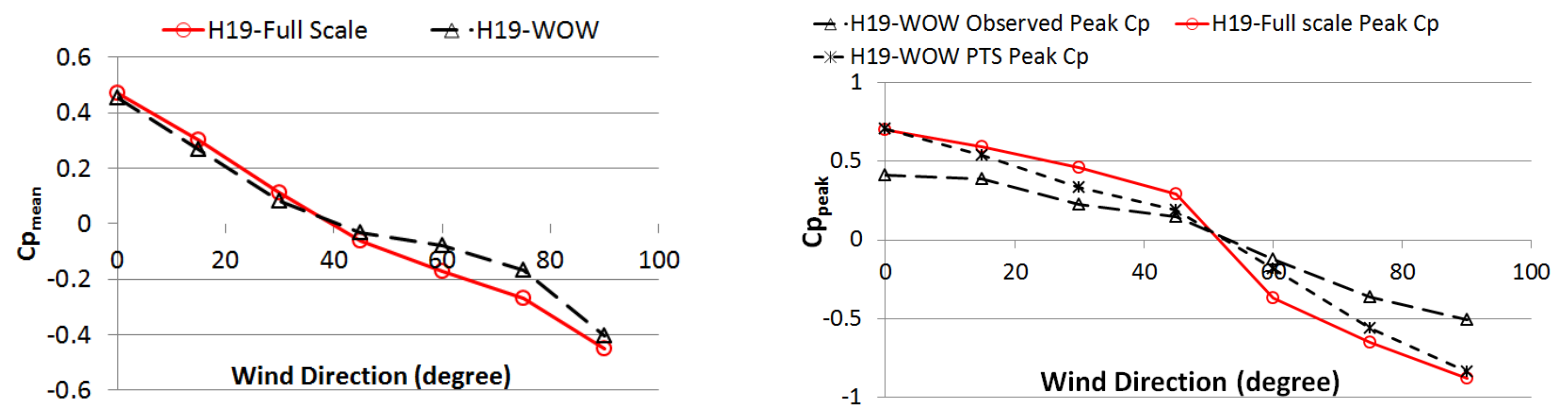

Fig.9. (Cont.). Silsoe cube wall Cp values comparisons vs. wind direction
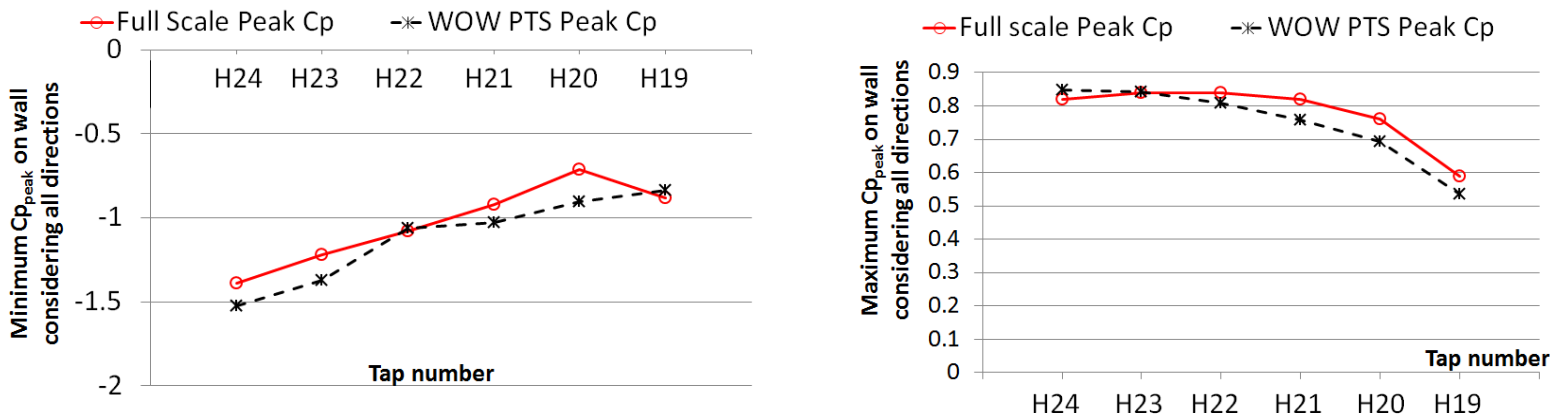

Fig. 10. Minimum and maximum of Silsoe cube wall $\mathrm{Cp}$ values comparisons considering all directions

Similar comparisons are shown in Fig. 11 for one selected tap (V7) experiencing the highest suctions seen on the roof. It can be seen that, while agreement is reasonably good for the angles creating highest suctions, there is a mismatch at 15 degrees between the data obtained from WOW and full scale. One reason for this might be that the lack of turbulence in WOW may have modified the process of formation of the roof corner vortex at this angle. More information on the other roof taps is provided below in the comparison of the PTS and 3DPTS methods. The PTS method did not provide such good predictions for quartering winds at taps in the center of the roof. This tended to occur when the suctions were not very high and, as discussed below, was largely resolved by using the 3DPTS method. Noteworthy was the fact that when attention was focused on the wind directions creating the highest suction for each roof tap the agreement of the PTS method with full scale was reasonably good, with a tendency for the PTS method to be conservative by between 0 and 15\%. This is demonstrated by the comparisons shown in Fig. 12 . 

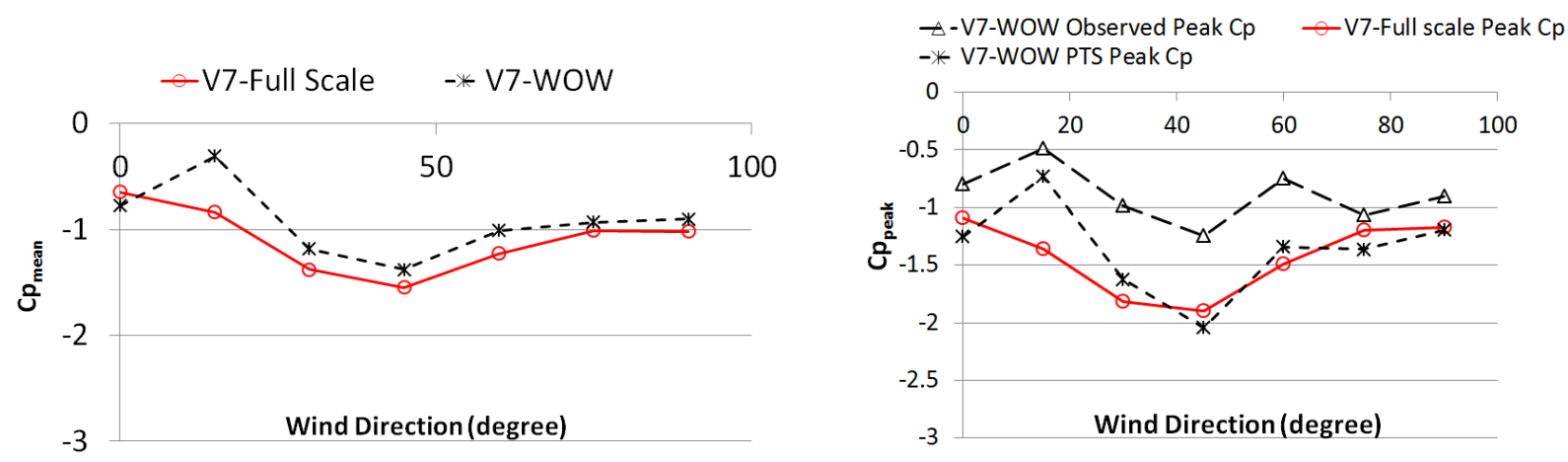

Fig.11. Roof Cp values comparisons vs. wind direction

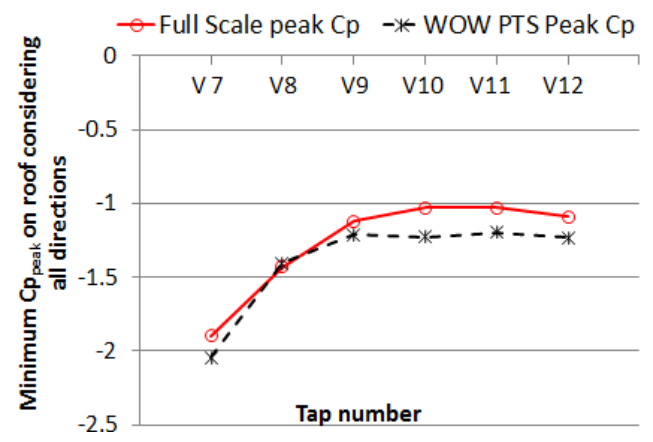

Fig.12. Minimum Roof Cp values comparisons for worst case direction of each tap

Figure 13 shows a comparison between the full scale data and the corrected pressure coefficient values roof and some selected wall pressure taps using the PTS and 3DPTS methods for the quartering wind direction, 45 degrees. For the roof pressure taps, it can be seen that the raw observed peak values were significantly low compared with full scale and application of the PTS procedure did not improve agreement by much except on tap V7 which saw the highest loading. Application of the 3DPTS method improved the results considerably. The reason for this is thought to be that at 45 degrees wind direction taps V8 through V12 saw relatively small peak suctions, but at angles either side of 45 degrees the suctions increased quite rapidly. Therefore any low frequency gusts that cause angles either side of 45 degrees to occur would cause high peaks to occur. In the 3DPTS procedure the influence of these adjacent angles and their higher peaks was taken into account. In this respect the 3DPTS procedure resembles an azimuthal weighted averaging process, which would tend to smooth out the peaks or troughs in the peak versus wind azimuth behavior predicted by the simpler PTS procedure. It should be noted that although reasonable agreement between the results of the 3DPTS method and full scale data was obtained for the pressure taps for which good full scale data 
was available, further validations are desirable for other wind directions for pressure taps in the critical regions near the roof corners where the vortices are smaller and more intense. Similar results were also observed for wall pressures i.e. the level of agreement between the corrected data using 3DPTS approach and the full scale data was higher than the PTS method. However, more relevant central directions would be 90 and 0 degrees when checking peak corner suctions on walls using 3DPTS method because those will produce high suctions. The primary objective of this paper was to examine the efficacy of the 3DPTS method to predict roof suctions for which 45 degrees wind direction serves well. It would be useful in future comparisons to undertake additional studies of negative pressures on wall taps using 3DPTS method at a central angle near 90 or 0 degrees.

(a)
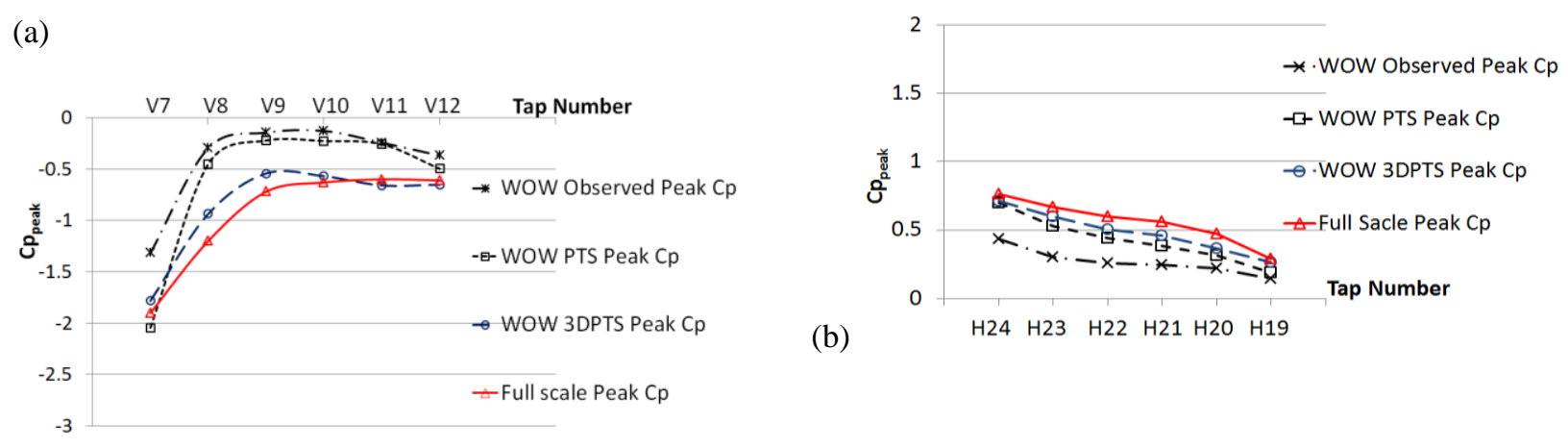

Fig.13. Peak Cp values at wind direction of 45 degrees: (a) Roof, b) Wall

\subsection{TTU pressure results}

The pressure coefficient comparisons presented in this section are based on full-scale TTU measurement results in which the pressure coefficients are defined in terms of the mean dynamic pressure as follows:

$C_{\text {mean }}=\frac{P_{\text {mean }}}{q_{\text {mean }}}$

$C_{\text {peak }}=\frac{P_{\text {peak }}}{q_{\text {mean }}}$

where $P_{\text {mean }}$ is the mean surface pressure, $P_{\text {peak }}$ is the highest positive or lowest negative pressure observed during the test duration at the TTU site and $q_{\text {mean }}$ is the mean dynamic pressure, $0.5 \rho U^{2}$, at roof height. The WOW mean and peak pressure data were obtained using the PTS and 3DPTS methods described in Sec. 2.6 and 2.7 respectively and were normalized in the same way as in Eqs. (62) and (63). Table 2 shows the test conditions for the TTU model in WOW and at Full-scale. 
Table 2. Test conditions for TTU model in WOW and at Full-scale

\begin{tabular}{lll}
\hline Test Characteristics & Full Scale & Model scale $\left(\boldsymbol{\lambda}_{\mathbf{L}}=\mathbf{1 / 6}\right)$ \\
\hline & $I_{u}=0.216$ & $I_{u H}=0.10$ \\
Turbulence intensity & $I_{v}=0.207$ & $I_{v H}=0.084$ \\
& $I_{w}=0.12$ & $I_{w H}=0.082$ \\
Integral length scale & $L_{u}^{x}=35 \mathrm{~m}^{*}$ & $L_{u}^{x}=0.43 \mathrm{~m}$ \\
Reference height & $Z_{r e f}=3.96 \mathrm{~m}$ & $Z_{\text {ref }}=0.66 \mathrm{~m}$ \\
Mean wind speed & $U_{F S}=7.66 \mathrm{~m} / \mathrm{s}$ & $U_{P S}=19.48$ \\
Test duration & $T_{F S}=15 \mathrm{~min}$ & $T_{P S}=2 \mathrm{~min}$ \\
Low pass filter frequency & $30 \mathrm{~Hz}$ & $141 \mathrm{~Hz}$ \\
\hline *Value was derived from ESDU (2001) at $3.96 \mathrm{~m}$ height for z0 $=0.05 \mathrm{~m}$ (open country)
\end{tabular}

Expected peak pressure coefficients were obtained with the WOW sample time divided into 80 subintervals. This means that each subinterval in model scale was equivalent to $120 / 80=1.5 \mathrm{sec}$. Based on the time scale, each subinterval at full scale was equivalent to $1.5 / 0.11=13.64 \mathrm{sec}$. So the targeted probability for the determination of the mode of the peak was $G=13.64 /(15 \times 60)=0.015$. For the present comparisons the expected peak pressure coefficients were compared, implying $G=1-0.57^{0.015}=0.0084$.

Figure 14 shows comparisons of the raw observed peak pressure coefficients on the roof of the TTU model, those processed using the 3DPTS method, and those measured at full scale (see Fig. $6 . b$ for the pressure tap notation). The results show that using 3DPTS substantially improves agreement with full scale compared with the raw observed peak values on the model. The best agreement with full scale in terms of percent difference tends to be obtained where the highest pressure coefficients occur. For some cases the corrected results obtained in WOW are higher than full scale data which suggest the appropriateness of use of WOW to test component strength, but not necessarily for all applications. Figure 15 shows a comparison between the peak pressure coefficients obtained using the PTS method, the 3DPTS method and full-scale data for the quartering wind direction, 45 degrees. The results showed that using the 3DPTS approach better agreement can be obtained with the full-scale data as compared with the PTS method. The difference is more pronounced for taps which are more sensitive to variations in wind direction. It should be noted that the 3DPTS procedure involves significant experimental effort in testing at small angle increments both in pitch and yaw angles about the target central angle. In this paper, 45 degrees azimuth and zero degrees pitch were selected as the representative target central angles for the purpose of assessing the methodology. The azimuth of 45 degrees for TTU does produce very high suctions seen under conical vortices (e.g. $\mathrm{Cp}=-18$ on tap A1) and so is a good representative test case for the purpose of assessing methodology. Other central angles may 
produce slightly higher overall suctions than what was observed for 45 degrees wind direction. These additional wind directions will be investigated in future.
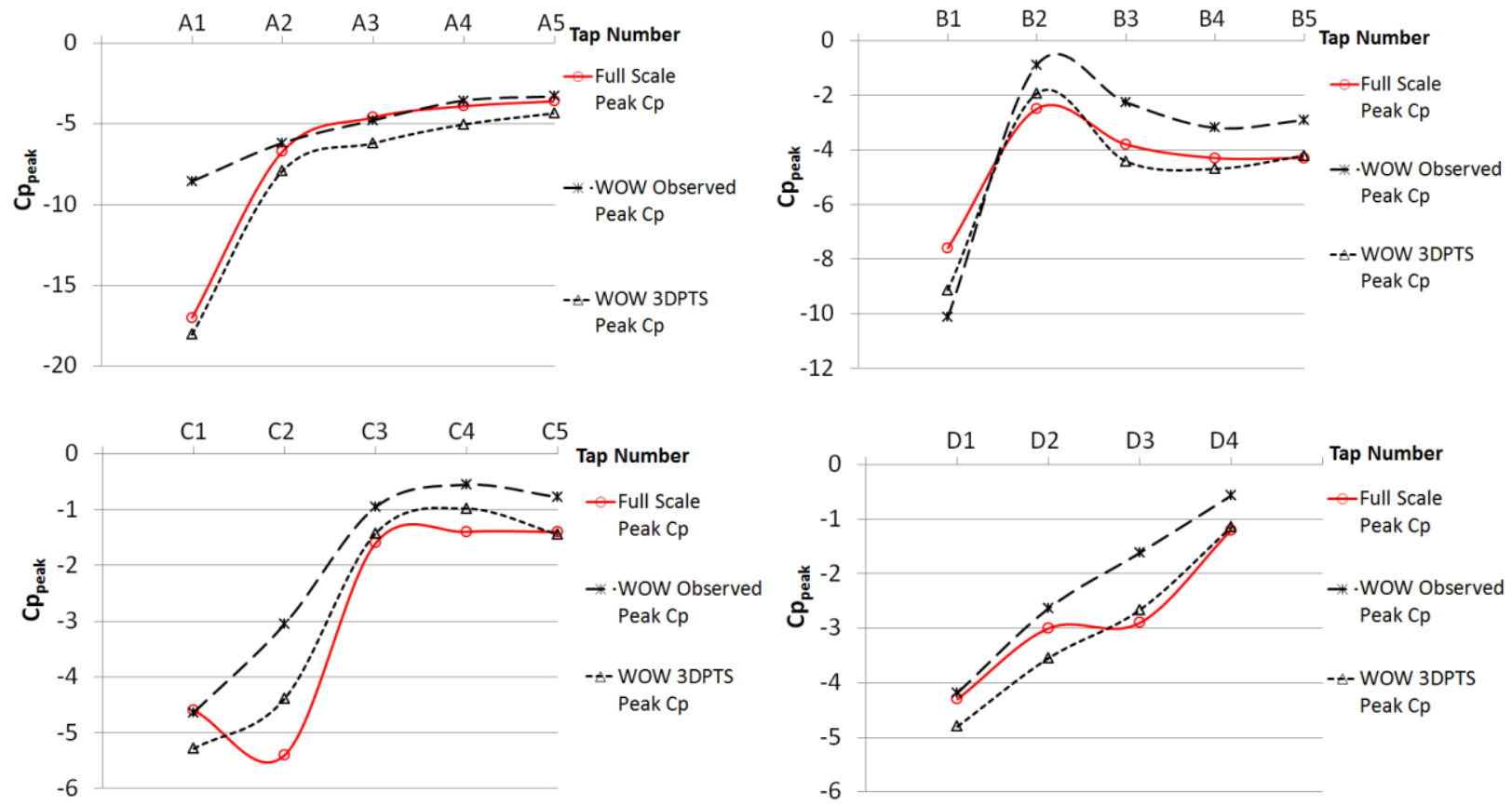

Fig.14. TTU Roof peak Cp values for 45 degrees wind direction
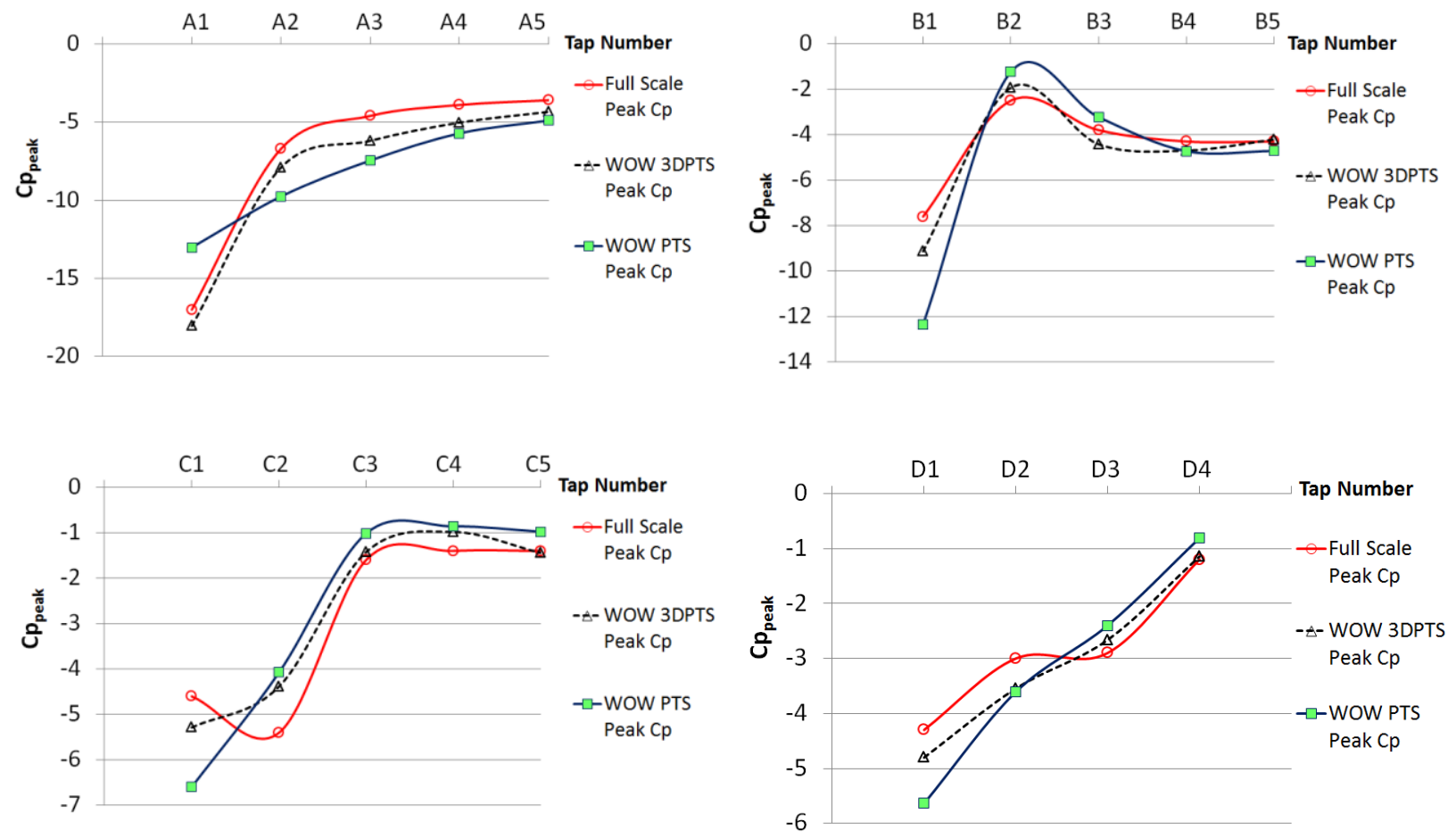

Fig.15. Comparison between PTS and 3DPTS method 


\section{Conclusions and Future work}

This paper describes a technique for testing and analyzing data from large scale models when only the high frequency end of the turbulence spectrum is simulated and for including low frequency effects using theoretical quasi-steady assumptions. The proposed test procedures and theoretical methods for including the effects of low frequency turbulence in post-test analysis have been assessed by comparing large scale model results obtained in the Wall of Wind facility at Florida International University for peak pressure coefficients with the full scale data from the Silsoe cube and TTU building. Two approaches for including the missing low frequency effects were developed: one, named here the PTS method, takes account of longitudinal low frequency fluctuations only; the other, called the 3DPTS method, includes also the effects of low frequency lateral and vertical turbulence. The results of the 3DPTS method agreed well enough with full scale peak pressure coefficients for both the Silsoe cube and the TTU buildings to encourage further investigations of the range of applicability of partial turbulence simulation methods. It is to be noted that application of 3DPTS producing conservative results (for testing material strengths) would permit the use of large scale models for certain types of testing. The PTS method, while simpler to apply, had a tendency to over predict suctions for wind directions where they were highest in magnitude and to under predict the suctions for wind directions where they were lowest. This behavior is what one would expect when the smoothing effect of low frequency lateral turbulence fluctuations is missing. However, when used to determine the highest pressure coefficient out of all wind directions, the PTS method appears to provide results that are conservative, but not excessively so. By accounting for low frequency turbulence effects analytically the proposed methodology allows significant relaxation of the scaling of turbulence integral scale, which is often a challenge when wind-tunnel testing small structures. This enables larger model scales to be employed, which has particular benefits for small structures and building components where testing at small scales such as 1:100 to 1:500 becomes impractical. The benefits of larger scale are improved geometric accuracy, enhanced spatial resolution of pressure taps, and potentially reduced Reynolds number effects. The proposed theory has the potential to substantially enhance the ability of existing test facilities such as the WOW and conventional boundary layer wind tunnels to make accurate predictions of full scale wind loads. The analysis method presented is still a work in progress. Some of the important cases, such as examining negative pressures on walls and investigating pressures in roof regions outside the corner vortex regions, will be examined in future. 
The present results, although limited to peak local pressures on the two test buildings for which good full scale data are available, are encouraging and invite further experimental efforts to explore the range of applicability the PTS method. In future it would be useful to apply the proposed partial turbulence technique to a wider range of model scales than in the present work and to compare with other benchmark aerodynamic data bases (such as NIST database). This would help to better define the range of applicability of the technique and to examine potential Reynolds number effects that could affect benchmark data from small scale tests. It should be emphasized that the although the present experiments were undertaken in the relatively large Wall of Wind facility, the PTS and 3DPTS techniques are equally applicable to conventional boundary layer wind tunnels.

\section{Acknowledgements}

The 12-fan Wall of Wind flow simulation and large-scale testing for this research was supported by the National Science Foundation (NSF) (NSF Award No. CMMI-1151003). We also acknowledge NSF support for Wall of Wind instrumentation (NSF Award No. CMMI-0923365). The Wall of Wind facility was partially funded by the Center of Excellence in Hurricane Damage Mitigation and Production Development through the FIU International Hurricane Research Center. We would like to acknowledge the help received from the PhD candidate, Ramtin Kargarmoakhar.

The help offered by the Wall of Wind lab manager Walter Conklin and research scientist Roy Liu is greatly acknowledged. We would also particularly like to thank Dr. Douglas Smith of Texas Tech University for kindly providing us with a large volume of the original data-set from the TTU full scale experiments.

\section{References}

ASCE/SEI 7-10, 2010. Minimum design loads for buildings and other structures. American Society of Civil Engineers, Reston, VA, USA.

ASCE/SEI 49-12, 2012. Wind Tunnel Testing for Buildings and Other Structures. American Society of Civil Engineers, Reston, VA, USA.

Asghari Mooneghi, M., 2014. Experimental and Analytical Methodologies for Predicting Peak Loads on Building Envelopes and Roofing Systems, FIU Electronic Theses and Dissertations. Paper 1846. http://digitalcommons.fiu.edu/etd/1846.

Asghari Mooneghi, M., Irwin, P., Gan Chowdhury, A., 2014. Large-scale testing on wind uplift of roof pavers. Journal of Wind Engineering and Industrial Aerodynamics 128, 22-36.

Asghari Mooneghi, M., Irwin, P.A., Gan Chowdhury, A., 2015. Partial Turbulence Simulation Method for Small Structures, 14th International Conference on Wind Engineering, Porto Alegre, Brazil.

Banks, D., 2011. Measuring peak wind loads on solar power assemblies, in: Proceedings of the The 13th International Conference on Wind Engineering. 
Chen, X., Kareem, A., Haan, F.L., 2000. Nonlinear aerodynamic analysis of bridges under turbulent winds: the new frontier in bridge aerodynamics, The International Conference on Advances in Structural Dynamics (ASD 2000), Hong Kong, China, pp. 475-482.

Cheung, J.C.K., Holmes, J.D., Melbourne, W.H., Lakshmanan, N., Bowditch, P., 1997. Pressures on a 110 scale model of the Texas Tech Building. Journal of wind engineering and industrial aerodynamics 69-71, 529-538.

Chu, C.R., Parlange, M.B., Katul, G.G., Albertson, J.D., 1996. Probability density functions of turbulent velocity and temperature in the atmospheric surface layer, Water Resources Research, pp. 1681-1688.

Cochran, L.S., Cermak, J.E., 1992. Full- and model-scale cladding pressures on the Texas Tech University experimental building. Journal of wind engineering and industrial aerodynamics 43, 15891600.

Davenport, A.G., King, J.P.C., 1984. Dynamic Forces on Long Span Bridges, 12th Congress of the International Association of Bridge and Structural Engineers (IABSE), Vancouver.

Diana, G., Cheli, F., Zasso, A., Bocciolone, M., 1999. Suspension bridge response to turbulent wind: Comparison of new numerical simulation method results with full scale data, in: Larose, Livesey (Eds.), Wind Engineering into the 21 Century, Balkema, Rotterdam, pp. 871-878.

ESDU, 2001. Characteristics of atmospheric turbulence near the ground. Part II single point data for strong winds (neutral atmosphere), Engineering Sciences Data Unit, Item 85020 Issued October 1985, with Amendments A to G August 2001.

Hillier, R., Cherry, N.J., 1981. The effects of stream turbulence on separation bubbles. Journal of wind engineering and industrial aerodynamics 8, 49-58.

Hutchins, N., Marusic, I., 2007. Large scale influences in near wall turbulence. Philosophical Transactions of the Royal Society A 365, $647-664$.

Irwin, P., 2009. Wind engineering research needs, building codes and project specific studies, in: Proceedings of the 11th Americas Conference on Wind Engineering.

Irwin, P., Cooper, K., Girard, R., 1979. Correction of distortion effects caused by tubing systems in measurements of fluctuating pressures. Journal of Wind Engineering and Industrial Aerodynamics 5, 93-107.

Irwin, P.A., 1981. A Simple Omnidirectional Sensor for Wind Tunnel Studies of Pedestrian Level Winds. Journal of Wind Engineering and Industrial Aerodynamics 7, 219-239.

Irwin, P.A., 1998. The role of wind tunnel modeling in the prediction of wind effects on bridges, in: Esdahl, A.L.S. (Ed.), The international symposium on advances in bridge aerodynamics, Copenhagen, Denmark, pp. 99-117.

Kargarmoakhar, R., Gan Chowdhury, A., Irwin, P., 2015. Reynolds number effects on twin box girder long span bridge aerodynamics. Wind and Structures 20, 327-347.

Kopp, G.A., Banks, D., 2013. Use of the wind tunnel test method for obtaining design wind loads on roof-mounted solar arrays. Journal of structural engineering 139, 284-287.

Kumar, K.S., Stathopoulos, T., 1998. Spectral Density Functions of Wind Pressures on Various Low Building Roof Geometries. Wind and Structures 1, 203-223.

Letchford, C.W., Mehta, K.C., 1993. The distribution and correlation of fluctuating pressures on the Texas Tech Building. Journal of wind engineering and industrial aerodynamics 50, 225-234.

Lin, J.X., Surry, D., Tieleman, H.W., 1995. The distribution of pressure near roof corners of flat roof low buildings. Journal of wind engineering and industrial aerodynamics 56, 235-265.

Lu, H., Porte-Agel, F., 2010. A modulated gradient model for large-eddy simulation: Application to a neutral atmospheric boundary layer. Physics of Fluids 22, 015109.

Macdonald, J.H.G., Irwin, P.A., Fletcher, M.S., 2002. Vortex-induced vibrations of the Second Severn Crossing cable-stayed bridge-full-scale and wind tunnel measurements. ICE: Structures and Buildings, 123-134.

Melbourne, W.H., 1980. Turbulence effects on maximum surface pressures - a mechanism and possibility of reduction. Wind Engineering, 541-551. 
Melbourne, W.H., 1993. Turbulence and the leading edge phenomenon. Journal of wind engineering and industrial aerodynamics 49, 45-63.

Okada, H., Ha, Y.-C., 1992. Comparison of wind tunnel and full-scale pressure measurement tests on the Texas Texh Building. Journal of wind engineering and industrial aerodynamics 43, 1601-1612.

Richards, P., Asghari Mooneghi, M., Gan Chowdhury, A., 2015. Combing Directionally Narrow Band Wind Loading Data in order to Match Wide Band Full-scale Situations, 14th International Conference on Wind Engineering, Porto Alegre, Brazil.

Richards, P.J., Hoxey, R.P., 2012. Pressures on a cubic building-Part 1: Full-scale results. Journal of wind engineering and industrial aerodynamics 102, 72-86.

Richards, P.J., Hoxey, R.P., Connell, B.D., Lander, D.P., 2007. Wind-tunnel modelling of the Silsoe Cube. Journal of Wind Engineering and Industrial Aerodynamics 95, 1384-1399.

Saathoff, P.J., Melbourne, W.H., 1989. The generation of peak pressures in separated/reattaching flows. Journal of wind engineering and industrial aerodynamics 32, 121-134.

Saathoff, P.J., Melbourne, W.H., 1997. Effects of free-stream turbulence on surface pressure fluctuation in a separation bubble. Journal Of Fluid Mechanics 337, 1-24.

Stathopoulos, T., Surry, D., 1983. Scale effects in wind tunnel testing of low buildings. Journal of wind engineering and industrial aerodynamics 13, 313-326.

Surry, D., 1991. Pressure measurements on the Texas tech building: Wind tunnel measurements and comparisons with full scale. Journal of wind engineering and industrial aerodynamics 38, 235-247.

Tieleman, H.W., 2003. Wind tunnel simulation of wind loading on low-rise structures: a review. Journal of wind engineering and industrial aerodynamics 91, 1627-1649.

Tieleman, H.W., Surry, D., Mehta, K.C., 1996. Full/model-scale comparison of surface pressures on the Texas Tech experimental building. Journal of wind engineering and industrial aerodynamics 61, 1-23.

Townsend, A.A., 1961. Equilibrium layers and wall turbulence. Journal of Fluid Mechanics 11, 97-120.

Wardlaw, R.L., Tanaka, H., Utsunomiya, H., 1983. Wind tunnel experiments on the effects of turbulence on the aerodynamic behaviour of bridge road decks. Journal of Wind Engineering and Industrial Aerodynamics 14, 247-257.

Wu, F., Sarkar, P.P., Mehta, K.C., Zhao, Z., 2001. Influence of incident wind turbulence on pressure fluctuations near flat-roof corners. Journal of wind engineering and industrial aerodynamics 89, 403420.

Yamada, H., Katsuchi, H., 2008. Wind-tunnel study on effects of small-scale turbulence on flow patterns around rectangular cylinder, 4th International Colloquium on Bluff Bodies Aerodynamics \& Applications, Italy.

Zhao, Z., 1997. Wind flow characteristics and their effects on low-rise buildings, Doctor of Philosophy Dissertation. Texas Tech University, Lubbock, Texas.

\section{Appendix A: Effective filter frequency for $\Delta t$ second moving average}

A variable $y$ that is fluctuating in the form of a sine wave obeys the relationship

$y=a \sin 2 \pi f t$

where $a=$ amplitude of the sinusoidal variations, $f=$ frequency of the wave in $\mathrm{Hz}$, and $t=$ time in seconds. If we sample the signal at rate $f_{s}$ then the $n^{\text {th }}$ sample will correspond to a time $=\frac{n}{f_{s}}$, and so

$y=a \sin 2 \pi \frac{f}{f_{s}} n$ 
It is shown in standard texts that the magnitude of the filter function corresponding to a moving average of $\mathrm{N}$ points of the sine wave is

$$
|H(f)|=\frac{\sin \pi \frac{f}{f_{S}} N}{N \sin \pi \frac{f}{f_{S}}}
$$

Since a time interval of $\Delta t$ corresponds to $N=\Delta t \times f_{s}$, then

$$
|H(\omega)|=\frac{\sin \pi f \Delta t}{f_{s} \Delta t \sin \left(\pi \frac{f}{f_{s}}\right)}
$$

If the sample rate $f_{s}$ is very high compared to the frequency of the sine wave, which would be true for the limiting the case of a continuous analogue signal, then this reduces to

$$
|H(\omega)|=\frac{\sin \pi f \Delta t}{\pi f \Delta t}
$$

In terms of the power spectrum the filter would be $|H|^{2}$. Figure A1 shows $|H|^{2}$ plotted against $f \Delta t$. It can be seen that the filter function is down to about a 0.5 value at $f \Delta t=0.45$. So, if we choose the half power level as being at our effective cut-off frequency, the effective cut off frequency is $f_{c}=\frac{0.45}{\Delta t}$. Or, viewing it the other way round, if $f_{c}=$ the cut-off frequency then the duration of the corresponding moving average is $\Delta t=\frac{0.45}{f_{c}}$. From this we see that a 3 -second moving average corresponds to a cut-off frequency of about $0.15 \mathrm{~Hz}$. Or a $1 \mathrm{~Hz}$ cut-off frequency corresponds to a moving 0.45 second average.

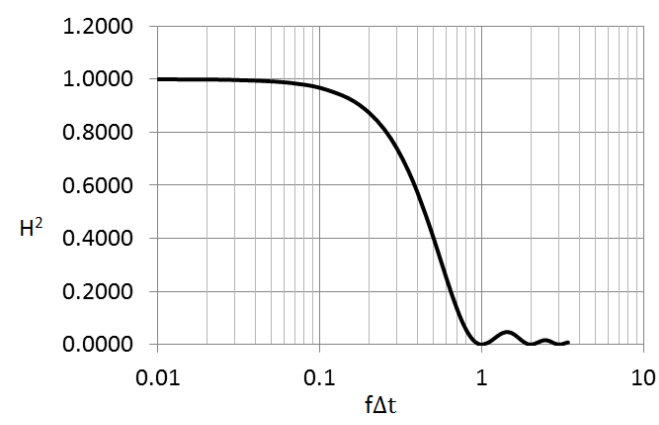

Fig. A1. Filter function for a moving average filter

In the PTS methodology developed in this paper, if the dividing frequency between the low and high frequencies $\left(n_{c}\right)$ be considered as a cut-off frequency in the context of the filter frequency for a $\Delta t$ second moving average, then Eq. (A6) below can be used for finding the equivalent gust-duration: 


$$
t_{\text {Gust }}=0.45 / n_{c}
$$

